\title{
OBLIQUE DUAL FUSION FRAMES
}

\author{
SIGRID B. HEINEKEN ${ }^{1, *}$ AND PATRICIA M. MORILLAS ${ }^{2}$ \\ ${ }^{1}$ Departamento de Matemática, FCEyN, Universidad de Buenos Aires, Pabellón I, Ciudad Universitaria, IMAS, \\ UBA-CONICET, C1428EGA C.A.B.A., Argentina \\ 2 Instituto de Matemática Aplicada San Luis, UNSL-CONICET and Departamento de Matemática, FCFMyN, \\ UNSL, Ejército de los Andes 950, 5700 San Luis, Argentina
}

\begin{abstract}
We introduce and develop the concept of oblique duality for fusion frames. This concept provides a mathematical framework to deal with problems in distributed signal processing where the signals, considered as elements in a Hilbert space and under certain consistency requirements, are analyzed in one subspace and are reconstructed in another subspace.
\end{abstract}

Key words: Frames, Oblique dual frames, Fusion frames, Oblique dual fusion frames, Consistent reconstruction, Oblique projections.

AMS subject classification: Primary 42C15; Secondary 42C40, 46C05, 47B10.

\section{INTRODUCTION}

Fusion frames [2, 3] (see also [1, Chapter 13]) generalize the notion of frames [1, 4, 13]. They are suitable in applications such as signal processing and sampling theory, in situations where one has to implement a local combination of data vectors. They allow representations of the elements of a separable Hilbert space using packets of linear coefficients.

Oblique dual frames have been introduced in 8 , and studied in 9, 10, 6, 5]. Oblique dual frames are useful in cases in which the analysis of a signal and its reconstruction have to be done in different subspaces.

\footnotetext{
* Corresponding author.
}

E-mail addresses: sheinek@dm.uba.ar (S. B. Heineken), morillas@unsl.edu.ar (P. M. Morillas). 
The aim of this paper is to introduce and develop the concept of oblique duality for fusion frames. This concept arises naturally from the notion of Eldar of oblique dual frames in [8] and our definition of dual fusion frames in [11, 12].

The paper is organized as follows. In Section 2 we give an overview of oblique projections, left inverses, frames, fusion frames and fusion frame systems.

We begin Section 3 introducing the concept of consistent reconstruction for fusion frames as a motivation of oblique duality. Then we introduce the definitions of oblique dual fusion frames and oblique dual fusion frame systems together with its basic properties. We present two classes of oblique dual fusion frames of special interest: the block-diagonal and, a subclass of them, the component preserving ones. The advantage of block diagonals is that they lead to a reconstruction formula that has a simpler expression. Oblique dual fusion frame systems are an example of this type. Component preserving duals are those that behave more similar to classical vectorial oblique duals, in particular, in the way that they can be obtained.

In Section 4 we analyze how the concepts of block-diagonal oblique dual frame, oblique dual fusion frame system and oblique dual frame are connected.

Section 5 presents statements that permit to obtain oblique dual fusion frames from dual fusion frames and viceversa.

Section 6 includes several results that give methods for constructing oblique dual fusion frames and oblique dual fusion frame systems. They provide different alternatives to select the most suitable from the computational point of view.

Finally, in Section 7 we introduce the concept of canonical oblique dual fusion frame. Some basic properties and a characterization are presented. Then we study when the canonical oblique dual is the unique dual and the existence of non-canonical oblique duals.

\section{Preliminaries}

We begin introducing some notation and then we briefly review definitions and properties that we use later.

2.1. Notation. We consider $\mathcal{H}, \mathcal{K}$ separable Hilbert spaces over $\mathbb{F}=\mathbb{R}$ or $\mathbb{F}=\mathbb{C}$. The space of bounded operators from $\mathcal{H}$ to $\mathcal{K}$ will be denoted by $L(\mathcal{H}, \mathcal{K})$ (we write $L(\mathcal{H})$ for $L(\mathcal{H}, \mathcal{H})$ ). For 
$T \in L(\mathcal{H}, \mathcal{K})$ we denote the image, the null space and the adjoint of $T$ by $\operatorname{Im}(T), \operatorname{Ker}(T)$ and $T^{*}$, respectively. If $T$ has closed range we also consider the Moore-Penrose pseudo-inverse of $T$ denoted by $T^{\dagger}$. The inner product and the norm in $\mathcal{H}$ will be denoted by $\langle\cdot, \cdot\rangle$ and $\|\cdot\|$, respectively. If $T \in L(\mathcal{H}, \mathcal{K})$, then $\|T\|_{F}$ and $\|T\|_{s p}$ denote the Frobenius and the spectral norms of $T$, respectively.

Let $I$ be a countable index set. If $\left\{\mathcal{H}_{i}\right\}_{i \in I}$ is a sequence of Hilbert spaces, we consider the Hilbert space

$$
\oplus_{i \in I} \mathcal{H}_{i}=\left\{\left(f_{i}\right)_{i \in I}: f_{i} \in \mathcal{H}_{i} \text { and }\left\{\left\|f_{i}\right\|\right\}_{i \in I} \in \ell^{2}(I)\right\}
$$

with inner product $\left\langle\left(f_{i}\right)_{i \in I},\left(g_{i}\right)_{i \in I}\right\rangle=\sum_{i \in I}\left\langle f_{i}, g_{i}\right\rangle$.

For $J \subseteq I$ let $\chi_{J}: I \rightarrow\{0,1\}$ be the characteristic function of $J$. We abbreviate $\chi_{\{j\}}=\chi_{j}$.

2.2. Oblique projections and left inverses. In the sequel $\mathcal{V}$ and $\mathcal{W}$ will be two closed subspaces of $\mathcal{H}$ such that $\mathcal{H}=\mathcal{V} \oplus \mathcal{W}^{\perp}$. By [6, Lemma 2.1] this is equivalent to $\mathcal{H}=\mathcal{W} \oplus \mathcal{V}^{\perp}$.

The oblique projection onto $\mathcal{V}$ along $\mathcal{W}^{\perp}$, is the unique operator that satisfies

$$
\begin{aligned}
& \pi_{\mathcal{V}, \mathcal{W}^{\perp}} f=f \text { for all } f \in \mathcal{V}, \\
& \pi_{\mathcal{V}, \mathcal{W}^{\perp}} f=0 \text { for all } f \in \mathcal{W}^{\perp} .
\end{aligned}
$$

Equivalently, $\operatorname{Im}\left(\pi_{\mathcal{V}, \mathcal{W}^{\perp}}\right)=\mathcal{V}$ and $\operatorname{Ker}\left(\pi_{\mathcal{V}, \mathcal{W}^{\perp}}\right)=\mathcal{W}^{\perp}$. If $\mathcal{W}=\mathcal{V}$ we obtain the orthogonal projection onto $\mathcal{W}$, which we denote by $\pi_{\mathcal{W}}$. The next properties involving oblique projections will be used throughout the paper.

Lemma 2.1. Let $\mathcal{V}$ and $\mathcal{W}$ be two closed subspaces of $\mathcal{H}$ such that $\mathcal{H}=\mathcal{V} \oplus \mathcal{W}^{\perp}$. Then

(1) $\pi_{\mathcal{V}, \mathcal{W}^{\perp}} \pi_{\mathcal{W}}=\pi_{\mathcal{V}, \mathcal{W}^{\perp}}$

(2) $\pi_{\mathcal{W}} \pi_{\mathcal{V}, \mathcal{W}^{\perp}}=\pi_{\mathcal{W}}$

Proof. (1) $\pi_{\mathcal{V}, \mathcal{W}^{\perp}} \pi_{\mathcal{W}}=\pi_{\mathcal{V}, \mathcal{W}^{\perp}}\left(\pi_{\mathcal{W}}+\pi_{\mathcal{W}^{\perp}}\right)=\pi_{\mathcal{V}, \mathcal{W}^{\perp}}$

(2) $\pi_{\mathcal{W}} \pi_{\mathcal{V}, \mathcal{W}^{\perp}}=\pi_{\mathcal{W}}\left(\pi_{\mathcal{V}, \mathcal{W}^{\perp}}+\pi_{\mathcal{W}^{\perp}, \mathcal{V}}\right)=\pi_{\mathcal{W}}$

Let $\mathcal{V}$ and $\mathcal{W}$ be two closed subspaces of $\mathcal{H}$ such that $\mathcal{H}=\mathcal{V} \oplus \mathcal{W}^{\perp}$. If $T \in L(\mathcal{H}, \mathcal{K})$ and $\operatorname{Ker}(T)=\mathcal{W}^{\perp}$, we denote by $\mathfrak{L}_{T}^{\mathcal{V}, \mathcal{W}^{\perp}}$ the set of oblique left inverses of $T$ on $\mathcal{V}$ along $\mathcal{W}^{\perp}$ which image is equal to $\mathcal{V}$, i. e.,

$$
\mathfrak{L}_{T}^{\mathcal{V}, \mathcal{W}^{\perp}}=\left\{U \in L(\mathcal{K}, \mathcal{H}): U T=\pi_{\mathcal{V}, \mathcal{W}^{\perp}} \text { and } \operatorname{Im}(U)=\mathcal{V}\right\} .
$$

The following lemma is useful to obtain oblique left inverses. 
Lemma 2.2. Let $\mathcal{V}$ and $\mathcal{W}$ be two closed subspaces of $\mathcal{H}$ such that $\mathcal{H}=\mathcal{V} \oplus \mathcal{W}^{\perp}$. Let $T \in L(\mathcal{H}, \mathcal{K})$ such that $\operatorname{Ker}(T)=\mathcal{W}^{\perp}$. Then $T_{\mid \mathcal{V}}$ is injective.

Proof. Let $f, g \in \mathcal{V}$ such that $T f=T g$. Since $\operatorname{Ker}(T)=\mathcal{W}^{\perp}$ the last equality is equivalent to $T \pi_{\mathcal{W}} f=T_{\mathcal{W}} g$. Since $T_{\mid \mathcal{W}}$ is injective, $\pi_{\mathcal{W}} f=\pi_{\mathcal{W}} g$. Therefore, $f-\pi_{\mathcal{W} \perp} f=g-\pi_{\mathcal{W} \perp} g$. Since $\mathcal{H}=\mathcal{V} \oplus \mathcal{W}^{\perp}, f=g$.

One way to get $U \in \mathfrak{L}_{T}^{\mathcal{V}, \mathcal{W}^{\perp}}$ is the following: By Lemma 2.2 if $g \in T(\mathcal{V})$ there exists a unique $f \in \mathcal{V}$ such that $T f=g$. Set $U g=f$. If $g \notin T(\mathcal{V})$ there are several possibilities, for example, $U g=U\left(g_{1}+g_{2}\right)=U g_{1}$ with $g_{1} \in T(\mathcal{V})$ and $g_{2} \in T(\mathcal{V})^{\perp}$.

If $\mathcal{V}=\mathcal{W}$, we write $\mathfrak{L}_{T}^{\mathcal{W}}=\left\{U \in L(\mathcal{K}, \mathcal{H}): U T=\pi_{\mathcal{W}}\right.$ and $\left.\operatorname{Im}(U)=\mathcal{W}\right\}$. This is the set of left inverses of $T$ on $\mathcal{W}$ such that $\operatorname{Im}(U)=\mathcal{W}$. The next proposition relates the sets $\mathfrak{L}_{T}^{\mathcal{W}}$ and $\mathfrak{L}_{T}^{\mathcal{V}, \mathcal{W}^{\perp}}$.

Proposition 2.3. Let $\mathcal{V}$ and $\mathcal{W}$ be two closed subspaces of $\mathcal{H}$ such that $\mathcal{H}=\mathcal{V} \oplus \mathcal{W}^{\perp}$. Let $T \in L(\mathcal{H}, \mathcal{K})$ such that $\operatorname{Ker}(T)=\mathcal{W}^{\perp}$. The map $A \in \mathfrak{L}_{T}^{\mathcal{W}} \mapsto \pi_{\mathcal{V}, \mathcal{W}} A \in \mathfrak{L}_{T}^{\mathcal{V}, \mathcal{W}^{\perp}}$ is a linear bijection, and its inverse is the map $B \in \mathfrak{L}_{T}^{\mathcal{V}, \mathcal{W}^{\perp}} \mapsto \pi_{\mathcal{W}} B \in \mathfrak{L}_{T}^{\mathcal{W}}$.

Proof. First we will show that the map $A \in \mathfrak{L}_{T}^{\mathcal{W}} \mapsto \pi_{\mathcal{V}, \mathcal{W} \perp} A \in \mathfrak{L}_{T}^{\mathcal{V}, \mathcal{W}^{\perp}}$ is well defined. Let $A \in \mathfrak{L}_{T}^{\mathcal{W}}$.

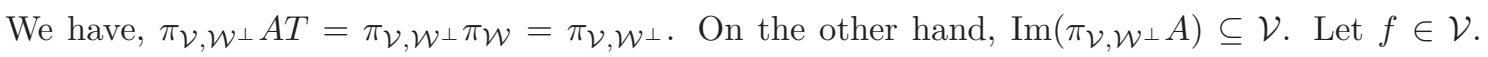
Since $\operatorname{Im}(A)=\mathcal{W}$ there exists $g \in \mathcal{K}$ such that $\pi_{\mathcal{W}} f=A g$ and then $f=\pi_{\mathcal{V}, \mathcal{W} \perp} f=\pi_{\mathcal{V}, \mathcal{W} \perp} \pi_{\mathcal{W}} f=$

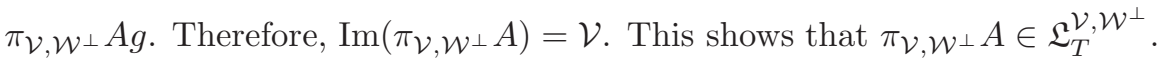

The linearity is trivial. Now we will prove that it is a bijection showing that the map $B \in$ $\mathfrak{L}_{T}^{\mathcal{V}, \mathcal{W}^{\perp}} \mapsto \pi_{\mathcal{W}} B \in \mathfrak{L}_{T}^{\mathcal{W}}$ is its inverse. First we note that in a similar manner as before it can be proved that it is a well defined linear map. Let $A \in \mathfrak{L}_{T}^{\mathcal{W}}$. Since $\operatorname{Im}(A)=\mathcal{W}, \pi_{\mathcal{W}} \pi_{\mathcal{V}, \mathcal{W}} A=\pi_{\mathcal{W}} A=$ $A$. Let now $B \in \mathfrak{L}_{T}^{\mathcal{V}, \mathcal{W}^{\perp}}$. Using that $\operatorname{Im}(B)=\mathcal{V}, \pi_{\mathcal{V}, \mathcal{W} \perp} \pi_{\mathcal{W}} B=\pi_{\mathcal{V}, \mathcal{W} \perp} B=B$. This proves that each map is the inverse of the other.

2.3. Frames. The concept of frame has been introduced by Duffin and Schaeffer in [7. Using a frame, each element of a Hilbert space has a representation which in general is not unique. This flexibility makes them attractive for many applications involving signal expansions.

We will now recall the concept of frame for a closed subspace of $\mathcal{H}$. 
Definition 2.4. Let $\mathcal{W}$ be a closed subspace of $\mathcal{H}$ and $\left\{f_{i}\right\}_{i \in I} \subset \mathcal{W}$. Then $\left\{f_{i}\right\}_{i \in I}$ is a frame for $\mathcal{W}$, if there exist constants $0<\alpha \leq \beta<\infty$ such that

$$
\alpha\|f\|^{2} \leq \sum_{i \in I}\left|\left\langle f, f_{i}\right\rangle\right|^{2} \leq \beta\|f\|^{2} \text { for all } f \in \mathcal{W} .
$$

If the right inequality in (2.1) is satisfied, $\left\{f_{i}\right\}_{i \in I}$ is a Bessel sequence for $\mathcal{W}$. The constants $\alpha$ and $\beta$ are the frame bounds. In case $\alpha=\beta$, we call $\left\{f_{i}\right\}_{i \in I}$ an $\alpha$-tight frame, and if $\alpha=\beta=1$ it is a Parseval frame for $\mathcal{W}$.

To a Bessel sequence $\mathcal{F}=\left\{f_{i}\right\}_{i \in I}$ for $\mathcal{W}$ we associate the synthesis operator

$$
T_{\mathcal{F}}: \ell^{2}(I) \rightarrow \mathcal{H}, T_{\mathcal{F}}\left\{c_{i}\right\}_{i \in I}=\sum_{i \in I} c_{i} f_{i},
$$

the analysis operator

$$
T_{\mathcal{F}}^{*}: \mathcal{H} \rightarrow \ell^{2}(I), T_{\mathcal{F}}^{*} f=\left\{\left\langle f, f_{i}\right\rangle\right\}_{i \in I},
$$

and the frame operator

$$
S_{\mathcal{F}}=T_{\mathcal{F}} T_{\mathcal{F}}^{*} .
$$

A Bessel sequence $\mathcal{F}=\left\{f_{i}\right\}_{i \in I}$ for $\mathcal{W}$ is a frame for $\mathcal{W}$ if and only $\operatorname{Im}\left(T_{\mathcal{F}}\right)=\mathcal{W}$, or equivalently, $S_{\mathcal{F}}$ is invertible when restricted to $\mathcal{W}$. Furthermore, $\mathcal{F}$ is an $\alpha$-tight frame for $\mathcal{W}$ if and only if $S_{\mathcal{F}}=\alpha \pi_{\mathcal{W}}$

If the subspace $\mathcal{W}$ is finite-dimensional we will consider finite frames for it, i.e., frames with a finite number of elements. It is worth to mention that if $\operatorname{dim}(\mathcal{W})<\infty$ then $\left\{f_{i}\right\}_{i \in I} \subset \mathcal{H}$ is a frame for $\mathcal{W}$ if and only $\operatorname{span}\left\{f_{i}\right\}_{i \in I}=\mathcal{W}$.

For more details about frames we refer the reader to 1, 4, 13, The concept of oblique dual frame [8, 9, 10, is defined as follows:

Definition 2.5. Let $\mathcal{W}$ and $\mathcal{V}$ be two closed subspaces of $\mathcal{H}$ such that $\mathcal{H}=\mathcal{V} \oplus \mathcal{W}^{\perp}$. Let $\mathcal{F}=\left\{f_{i}\right\}_{i \in I}$ be a frame for $\mathcal{W}$ and $\mathcal{G}=\left\{g_{i}\right\}_{i \in I}$ be a frame for $\mathcal{V}$. If

$$
T_{\mathcal{G}} T_{\mathcal{F}}^{*}=\pi_{\mathcal{V}, \mathcal{W}^{\perp}}
$$

we say that $\mathcal{G}$ is an oblique dual frame of $\mathcal{F}$ on $\mathcal{V}$.

The sequence $\left\{\pi_{\mathcal{V}, \mathcal{W}^{\perp}} S_{\mathcal{F}}^{\dagger} f_{i}\right\}_{i \in I}$ is the canonical oblique dual frame of $\left\{f_{i}\right\}_{i \in I}$ on $\mathcal{V}$.

Remark 2.6. When $\mathcal{V}=\mathcal{W}$ we obtain the classical duals and we simply say dual frame instead of oblique dual frame on $\mathcal{W}$. 
A Riesz basis for $\mathcal{W}$ is a frame for $\mathcal{W}$ which is also a basis. Observe that a Riesz basis has a unique dual, the canonical one.

2.4. Fusion frames. Fusion frames were introduced by Casazza and Kutyniok in [2] under the name of frames of subspaces. They turned out to be a useful tool for handling problems in sensor networking, distributed processing, etc. Throughout the paper we will work with fusion frames for closed subspaces of $\mathcal{H}$.

Assume $\left\{W_{i}\right\}_{i \in I}$ is a family of closed subspaces in $\mathcal{W}$, and $\left\{w_{i}\right\}_{i \in I}$ a family of weights, i.e., $w_{i}>0$ for all $i \in I$. We denote $\left\{W_{i}\right\}_{i \in I}$ with $\mathbf{W},\left\{w_{i}\right\}_{i \in I}$ with $\mathbf{w}$ and $\left\{\left(W_{i}, w_{i}\right)\right\}_{i \in I}$ with $(\mathbf{W}, \mathbf{w})$. If $T \in L(\mathcal{H}, \mathcal{K})$ we write $(T \mathbf{W}, \mathbf{w})$ for $\left\{\left(T W_{i}, w_{i}\right)\right\}_{i \in I}$.

We consider the Hilbert space $\mathcal{K}_{\mathcal{W}}:=\oplus_{i \in I} W_{i}$.

Definition 2.7. We say that $(\mathbf{W}, \mathbf{w})$ is a fusion frame for $\mathcal{W}$, if there exist constants $0<\alpha \leq$ $\beta<\infty$ such that

$$
\alpha\|f\|^{2} \leq \sum_{i \in I} w_{i}^{2}\left\|\pi_{W_{i}}(f)\right\|^{2} \leq \beta\|f\|^{2} \text { for all } f \in \mathcal{W} .
$$

We call $\alpha$ and $\beta$ the fusion frame bounds. The family $(\mathbf{W}, \mathbf{w})$ is called an $\alpha$-tight fusion frame for $\mathcal{W}$, if in (2.2) the constants $\alpha$ and $\beta$ can be chosen so that $\alpha=\beta$, and a Parseval fusion frame for $\mathcal{W}$ provided that $\alpha=\beta=1$. If $(\mathbf{W}, \mathbf{w})$ has an upper fusion frame bound, but not necessarily a lower bound, it is called a Bessel fusion sequence for $\mathcal{W}$ with Bessel fusion bound $\beta$. If $w_{i}=c$ for all $i \in I$, we write $\mathbf{w}=c$. If $\mathcal{W}$ is the direct sum of the $W_{i}$ we say that $(\mathbf{W}, \mathbf{w})$ is a Riesz fusion basis for $\mathcal{W}$. We will refer to a fusion frame that is not a Riesz fusion basis as an overcomplete fusion frame. A fusion frame $(\mathbf{W}, 1)$ is an orthonormal fusion basis for $\mathcal{W}$ if $\mathcal{W}$ is the orthogonal sum of the subspaces $W_{i}$.

To a Bessel fusion sequence $(\mathbf{W}, \mathbf{w})$ for $\mathcal{W}$ we associate the synthesis operator

$$
T_{\mathbf{W}, \mathbf{w}}: \mathcal{K}_{\mathcal{W}} \rightarrow \mathcal{H}, \quad T_{\mathbf{W}, \mathbf{w}}\left(f_{i}\right)_{i \in I}=\sum_{i \in I} w_{i} f_{i},
$$

the analysis operator

$$
T_{\mathbf{W}, \mathbf{w}}^{*}: \mathcal{H} \rightarrow \mathcal{K}_{\mathcal{W}}, \quad T_{\mathbf{W}, \mathbf{w}}^{*} f=\left(w_{i} \pi_{W_{i}}(f)\right)_{i \in I}
$$

and the fusion frame operator

$$
S_{\mathbf{W}, \mathbf{w}}=T_{\mathbf{W}, \mathbf{w}} T_{\mathbf{W}, \mathbf{w}}^{*} .
$$


As it happens for frames, $(\mathbf{W}, \mathbf{w})$ is a Bessel fusion sequence for $\mathcal{W}$ if and only if $T_{\mathbf{W}, \mathbf{w}}$ is a well defined bounded linear operator. A Bessel fusion sequence $(\mathbf{W}, \mathbf{w})$ for $\mathcal{W}$ is a fusion frame for $\mathcal{W}$ if and only if $\operatorname{Im}\left(T_{\mathbf{W}, \mathbf{w}}\right)=\mathcal{W}$, or equivalently, $S_{\mathbf{W}, \mathbf{w}}$ restricted to $\mathcal{W}$ is bijective. Additionally, $(\mathbf{W}, \mathbf{w})$ is an $\alpha$-tight fusion frame for $\mathcal{W}$ if and only if $S_{\mathbf{W}, \mathbf{w}}=\alpha \pi_{\mathcal{W}}$.

For finite-dimensional subspaces $\mathcal{W}$ we will consider finite fusion frames, i.e., fusion frames with a finite set of indices. Note that if $\operatorname{dim}(\mathcal{W})<\infty$ then $(\mathbf{W}, \mathbf{w})$ is a frame for $\mathcal{W}$ if and only $\operatorname{span} \cup_{i \in I} W_{i}=\mathcal{W}$.

Having fusion frames allows local processing in each of the subspaces. In view of this, having a set of local frames for its subspaces is convenient.

Definition 2.8. Let $\mathcal{W}$ be a closed subspace of $\mathcal{H}$, let $(\mathbf{W}, \mathbf{w})$ be a fusion frame (Bessel fusion sequence $)$ for $\mathcal{W}$, and let $\left\{f_{i, l}\right\}_{l \in L_{i}}$ be a frame for $W_{i}$ for $i \in I$. Then $\left\{\left(W_{i}, w_{i},\left\{f_{i, l}\right\}_{l \in L_{i}}\right)\right\}_{i \in I}$ is called a fusion frame system (Bessel fusion system) for $\mathcal{W}$.

Throughout this work we will use the notation $\mathcal{F}_{i}=\left\{f_{i, l}\right\}_{l \in L_{i}}, \mathcal{F}=\left\{\mathcal{F}_{i}\right\}_{i \in I}, \mathbf{w} \mathcal{F}=\left\{w_{i} \mathcal{F}_{i}\right\}_{i \in I}$, and we write $(\mathbf{W}, \mathbf{w}, \mathcal{F})$ for $\left\{\left(W_{i}, w_{i},\left\{f_{i, l}\right\}_{l \in L_{i}}\right)\right\}_{i \in I}$. If $T \in L(\mathcal{H}, \mathcal{K})$ we write $T \mathcal{F}$ for $\left\{\left\{T f_{i, l}\right\}_{l \in L_{i}}\right\}_{i \in I}$ and $T \mathcal{F}_{i}$ for $\left\{T f_{i, l}\right\}_{l \in L_{i}}$.

Theorem 2.9. 2, Theorem 3.2] Let $\mathcal{W}$ be a closed subspace of $\mathcal{H}$. Given $(\mathbf{W}, \mathbf{w})$, let $\mathcal{F}_{i}$ be a frame for $W_{i}$ with frame bounds $\alpha_{i}, \beta_{i}$ for each $i \in I$ such that $0<\alpha=\inf _{i \in I} \alpha_{i} \leq \sup _{i \in I} \beta_{i}=\beta<\infty$. The following assertions are equivalents:

$(1) \mathbf{w} \mathcal{F}$ is a frame for $\mathcal{W}$.

(2) $(\mathbf{W}, \mathbf{w})$ is a fusion frame for $\mathcal{W}$.

If $(\mathbf{W}, \mathbf{w})$ is a fusion frame for $\mathcal{W}$ with fusion frame bounds $\gamma$ and $\delta$, then $\mathbf{w} \mathcal{F}$ is a frame for $\mathcal{W}$ with frame bounds $\alpha \gamma$ and $\beta \delta$. If $\mathbf{w} \mathcal{F}$ is a frame for $\mathcal{W}$ with frame bounds $\gamma$ and $\delta$, then $(\mathbf{W}, \mathbf{w})$ is a fusion frame for $\mathcal{W}$ with fusion frame bounds $\frac{\gamma}{\beta}$ and $\frac{\delta}{\alpha}$.

The previous assertions are valid if we replace fusion frame by Bessel fusion sequence and consider only the upper bounds.

For more details about fusion frames and fusion frame systems we refer the reader to [2, 3] (see also [1, Chapter 13]). 


\section{OBlique DUALITY FOR FUSION FRAMES AND FUSION FRAME SYSTEMS}

One reason for considering oblique duality is the so called consistent reconstruction. Based on the vectorial case [8, 10] and the relation between frames and fusion frames, we next introduce the concept of consistent reconstruction for fusion frames.

3.1. Consistent reconstruction. Let $\mathcal{W}$ and $\mathcal{V}$ be two closed subspaces of $\mathcal{H}$. Let $(\mathbf{W}, \mathbf{w})$ be a fusion frame for $\mathcal{W}$. Assume that the measurements $T_{\mathbf{W}, \mathbf{w}}^{*} f=\left(w_{i} \pi_{W_{i}} f\right)_{i \in I}$ of an unknown signal $f \in \mathcal{H}$ are given. Our goal is the reconstruction of $f$ from these measurements using a fusion frame $(\mathbf{V}, \mathbf{v})$ for $\mathcal{V}$ in such a way that the reconstruction $\widehat{f}$ is a good approximation of $f$. Specifically the following two conditions are required:

(i) Uniqueness of the reconstructed signal: If $f, g \in \mathcal{V}$ and $T_{\mathbf{W}, \mathbf{w}}^{*} f=T_{\mathbf{W}, \mathbf{w}}^{*} g$, then $f=g$.

(ii) Consistent sampling: $T_{\mathbf{W}, \mathbf{w}}^{*} \widehat{f}=T_{\mathbf{W}, \mathbf{w}}^{*} f$ for all $f \in \mathcal{H}$.

Requirement (i) is equivalent to $\mathcal{V} \cap \mathcal{W}^{\perp}=\{0\}$. To see this, suppose that (i) holds and consider $f \in \mathcal{V} \cap \mathcal{W}^{\perp}$. Since $f \in \mathcal{W}^{\perp}, T_{\mathbf{W}, \mathbf{w}}^{*} f=0=T_{\mathbf{W}, \mathbf{w}}^{*} 0$. Since $f \in \mathcal{V}$, by (i) this implies that $f=0$, and thus $\mathcal{V} \cap \mathcal{W}^{\perp}=\{0\}$. Suppose now that $\mathcal{V} \cap \mathcal{W}^{\perp}=\{0\}$. Let $f, g \in \mathcal{V}$ such that $T_{\mathbf{W}, \mathbf{w}}^{*}(f-g)=0$. Thus $f-g \in \operatorname{Ker}\left(T_{\mathbf{W}, \mathbf{w}}^{*}\right)=\operatorname{Im}\left(T_{\mathbf{W}, \mathbf{w}}\right)^{\perp}=\mathcal{W}^{\perp}$. Consequenlty, $f-g=0$. Therefore, (i) holds.

In case that (ii) is satisfied we say that $\widehat{f} \in \mathcal{V}$ is a consistent reconstruction of $f \in \mathcal{H}$ on $\mathcal{V}$ along $\mathcal{W}^{\perp}$.

From (i) and (ii), we deduce that if $f \in \mathcal{V}$ then $\widehat{f}=f$. So in this case, $f$ can be perfectly reconstructed.

The next result shows that consistent reconstruction is linked to oblique projections.

Theorem 3.1. Let $\mathcal{W}$ and $\mathcal{V}$ be two closed subspaces of $\mathcal{H}$ such that $\mathcal{H}=\mathcal{V} \oplus \mathcal{W}^{\perp}$. Let $(\mathbf{W}, \mathbf{w})$ be a fusion frame for $\mathcal{W}$. Then $\widehat{f} \in \mathcal{V}$ is a consistent reconstruction of $f \in \mathcal{H}$ if and only if $\widehat{f}=\pi_{\mathcal{V}, \mathcal{W}^{\perp}} f$.

Proof. Suppose that $\widehat{f} \in \mathcal{V}$ is a consistent reconstruction of $f \in \mathcal{W}$, i.e. (ii) holds. Then $\widehat{f}-f \in$ $\operatorname{Ker}\left(T_{\mathbf{W}, \mathbf{w}}^{*}\right)=\operatorname{Im}\left(T_{\mathbf{W}, \mathbf{w}}\right)^{\perp}=\mathcal{W}^{\perp}$. Thus $f=\widehat{f}+(f-\widehat{f})$ where $\widehat{f} \in \mathcal{V}$ and $\widehat{f}-f \in \mathcal{W}^{\perp}$. Taking into account that $\mathcal{H}=\mathcal{V} \oplus \mathcal{W}^{\perp}$, this implies that $\widehat{f}=\pi_{\mathcal{V}, \mathcal{W}^{\perp}} f$ and $\widehat{f}-f=\pi_{\mathcal{W}^{\perp}, \mathcal{V}} f$.

Assume now that $\widehat{f}=\pi_{\mathcal{V}, \mathcal{W}^{\perp}} f$. Since $\mathcal{H}=\mathcal{V} \oplus \mathcal{W}^{\perp}, \widehat{f}-f=\pi_{\mathcal{W}^{\perp}, \mathcal{V}} f \in \mathcal{W}^{\perp}=\operatorname{Im}\left(T_{\mathbf{W}, \mathbf{w}}\right)^{\perp}=$ $\operatorname{Ker}\left(T_{\mathbf{W}, \mathbf{w}}^{*}\right)$. Therefore $T_{\mathbf{W}, \mathbf{w}}^{*}(\widehat{f}-f)=0$ and (ii) holds. 
3.2. Oblique dual fusion frames. In [11, 12 the concepts of dual fusion frame and dual fusion frames system are introduced and studied. Motivated by these concepts and by Definition 2.5 we introduce now the definition of oblique dual fusion frame and later the one of oblique dual fusion frame system.

Definition 3.2. Let $\mathcal{W}$ and $\mathcal{V}$ be two closed subspaces of $\mathcal{H}$ such that $\mathcal{H}=\mathcal{V} \oplus \mathcal{W}^{\perp}$. Let $(\mathbf{W}, \mathbf{w})$ be a fusion frame for $\mathcal{W}$ and $(\mathbf{V}, \mathbf{v})$ be a fusion frame for $\mathcal{V}$. We say that $(\mathbf{V}, \mathbf{v})$ is an oblique dual fusion frame of $(\mathbf{W}, \mathbf{w})$ on $\mathcal{V}$ if there exists $Q \in L\left(K_{\mathcal{W}}, K_{\mathcal{V}}\right)$ such that

$$
T_{\mathbf{V}, \mathbf{v}} Q T_{\mathbf{W}, \mathbf{w}}^{*}=\pi_{\mathcal{V}, \mathcal{W}^{\perp}}
$$

The operator $Q$ is actually important in the definition. If we need to do an explicit reference to it we say that $(\mathbf{V}, \mathbf{v})$ is a $Q$-oblique dual fusion frame of $(\mathbf{W}, \mathbf{w})$. Note that if $(\mathbf{V}, \mathbf{v})$ is a $Q$-oblique dual fusion frame of $(\mathbf{W}, \mathbf{w})$ on $\mathcal{V}$, then $(\mathbf{W}, \mathbf{w})$ is a $Q^{*}$-oblique dual fusion frame of $(\mathbf{V}, \mathbf{v})$ on $\mathcal{W}$. As we will see in Lemma 3.4 Bessel fusion sequences $(\mathbf{W}, \mathbf{w})$ for $\mathcal{W}$ and $(\mathbf{V}, \mathbf{v})$ for $\mathcal{V}$ that satisfy (3.1), are automatically fusion frames.

As a consequence of Definition 3.2 and Theorem 3.1, we obtain the following result which establishes that oblique duality yields consistent reconstruction.

Corollary 3.3. Let $\mathcal{W}$ and $\mathcal{V}$ be two closed subspaces of $\mathcal{H}$ such that $\mathcal{H}=\mathcal{V} \oplus \mathcal{W}^{\perp}$. Let $(\mathbf{W}, \mathbf{w})$ be a fusion frame for $\mathcal{W},(\mathbf{V}, \mathbf{v})$ be a fusion frame for $\mathcal{V}$ and $Q \in L\left(K_{\mathcal{W}}, K_{\mathcal{V}}\right)$. Then $\widehat{f}:=T_{\mathbf{V}, \mathbf{v}} Q T_{\mathbf{W}, \mathbf{w}}^{*} f$ is a consistent reconstruction of $f$ for all $f \in \mathcal{H}$ if and only if $(\mathbf{V}, \mathbf{v})$ is a $Q$-oblique dual fusion frame of $(\mathbf{W}, \mathbf{w})$ on $\mathcal{V}$.

It is worth to mention that one reason to introduce first a general class of oblique dual fusion frames as in Definition 3.2, requiring only boundedness of the operator $Q$, is to ask for the minimal conditions needed to obtain the different desired properties for oblique dual fusion frames. In particular, for this general class we have consistent reconstruction as Corollary 3.3 shows and the following lemma, which generalizes the basic properties that are valid for dual and oblique dual frames. It is analogous to [11, Lemma 3.2] and gives equivalent conditions for two Bessel fusion sequences to be oblique dual fusion frames. 
Lemma 3.4. Let $\mathcal{W}$ and $\mathcal{V}$ be two closed subspaces of $\mathcal{H}$ such that $\mathcal{H}=\mathcal{V} \oplus \mathcal{W}^{\perp}$. Let $(\mathbf{W}, \mathbf{w})$ be a Bessel fusion sequence for $\mathcal{W},(\mathbf{V}, \mathbf{v})$ be a Bessel fusion sequence for $\mathcal{V}$, and let $Q \in L\left(K_{\mathcal{W}}, K_{\mathcal{V}}\right)$. Then the following statements are equivalent:

(1) $T_{\mathbf{V}, \mathbf{v}} Q T_{\mathbf{W}, \mathbf{w}}^{*} f=f$ for all $f \in \mathcal{V}$.

(2) $T_{\mathbf{W}, \mathbf{w}} Q^{*} T_{\mathbf{V}, \mathbf{v}}^{*} f=f$ for all $f \in \mathcal{W}$.

(3) $\pi_{\mathcal{V}, \mathcal{W}^{\perp}} f=T_{\mathbf{V}, \mathbf{v}} Q T_{\mathbf{W}, \mathbf{w}}^{*} f$ for all $f \in \mathcal{H}$.

(4) $\pi_{\mathcal{W}, \mathcal{V} \perp} f=T_{\mathbf{W}, \mathbf{w}} Q^{*} T_{\mathbf{V}, \mathbf{v}}^{*} f$ for all $f \in \mathcal{H}$.

(5) $\left\langle\pi_{\mathcal{W}, \mathcal{V}^{\perp}} f, g\right\rangle=\left\langle Q^{*} T_{\mathbf{V}, \mathbf{v}}^{*} f, T_{\mathbf{W}, \mathbf{w}}^{*} g\right\rangle$ for all $f, g \in \mathcal{H}$.

(6) $\left\langle\pi_{\mathcal{V}, \mathcal{W}^{\perp}} f, g\right\rangle=\left\langle Q T_{\mathbf{W}, \mathbf{w}}^{*} f, T_{\mathbf{V}, \mathbf{v}}^{*} g\right\rangle$ for all $f, g \in \mathcal{H}$.

(7) $T_{\mathbf{W}, \mathbf{w}}^{*} \mid \mathcal{V}$ is injective, $T_{\mathbf{V}, \mathbf{v}} Q$ is surjective and $\left(T_{\mathbf{W}, \mathbf{w}}^{*} T_{\mathbf{V}, \mathbf{v}} Q\right)^{2}=T_{\mathbf{W}, \mathbf{w}}^{*} T_{\mathbf{V}, \mathbf{v}} Q$.

(8) $T_{\mathbf{V}, \mathbf{v}}^{*} \mid \mathcal{W}$ is injective, $T_{\mathbf{W}, \mathbf{w}} Q^{*}$ is surjective and $\left(T_{\mathbf{V}, \mathbf{v}}^{*} T_{\mathbf{W}, \mathbf{w}} Q^{*}\right)^{2}=T_{\mathbf{V}, \mathbf{v}}^{*} T_{\mathbf{W}, \mathbf{w}} Q^{*}$.

In case any of these equivalent conditions are satisfied, $(\mathbf{W}, \mathbf{w})$ is a fusion frame for $\mathcal{W},(\mathbf{V}, \mathbf{v})$ is a fusion frame for $\mathcal{V},(\mathbf{V}, \mathbf{v})$ is a $Q$-oblique dual fusion frame of $(\mathbf{W}, \mathbf{w})$ on $\mathcal{V}$, and $(\mathbf{W}, \mathbf{w})$ is an oblique $Q^{*}$-dual fusion frame of $(\mathbf{V}, \mathbf{v})$ on $\mathcal{W}$.

Proof. $(1) \Leftrightarrow(3)$ and $(2) \Leftrightarrow(3)$ are immediate.

$(3) \Rightarrow(4): T_{\mathbf{V}, \mathbf{v}} Q T_{\mathbf{W}, \mathbf{w}}^{*}=\pi_{\mathcal{V}, \mathcal{W}^{\perp}}$. Taking adjoint $T_{\mathbf{W}, \mathbf{w}} Q^{*} T_{\mathbf{V}, \mathbf{v}}^{*}=\pi_{\mathcal{V}, \mathcal{W}^{\perp}}$. But $\pi_{\mathcal{V}, \mathcal{W}^{\perp}}^{*}=\pi_{\mathcal{W}, \mathcal{V}^{\perp}}$ hence (3) follows.

$(4) \Rightarrow(5)$ is clear as well as $(3) \Rightarrow(6)$.

$(5) \Rightarrow(4)$ : For $f \in \mathcal{H}, T_{\mathbf{W}, \mathbf{w}} Q^{*} T_{\mathbf{V}, \mathbf{v}}^{*} f=T_{\mathbf{W}, \mathbf{w}} Q^{*} T_{\mathbf{V}, \mathbf{v}}^{*} \pi_{\mathcal{V}} f$ is well defined since $(\mathbf{W}, \mathbf{w})$ is a Bessel fusion sequence for $\mathcal{W}$ and $(\mathbf{V}, \mathbf{v})$ is a Bessel fusion sequence for $\mathcal{V}$. By $(5)$,

$$
\left\langle\pi_{\mathcal{W}, \mathcal{V}^{\perp}} f-T_{\mathbf{W}, \mathbf{w}} Q^{*} T_{\mathbf{V}, \mathbf{v}}^{*} f, g\right\rangle=0 \text { for all } g \in \mathcal{H}
$$

and so we obtain (4). Analogously $(6) \Rightarrow(3)$.

$(1) \Leftrightarrow(7)$ : By (1), $T_{\mathbf{W}, \mathbf{w}}^{*} \mid \mathcal{V}$ is injective, $T_{\mathbf{V}, \mathbf{v}} Q$ is surjective and

$$
\left(T_{\mathbf{W}, \mathbf{w}}^{*} T_{\mathbf{V}, \mathbf{v}} Q\right)^{2}=T_{\mathbf{W}, \mathbf{w}}^{*}\left(T_{\mathbf{V}, \mathbf{v}} Q T_{\mathbf{W}, \mathbf{w}}^{*}\right) T_{\mathbf{V}, \mathbf{v}} Q=T_{\mathbf{W}, \mathbf{w}}^{*} T_{\mathbf{V}, \mathbf{v}} Q .
$$

$(7) \Rightarrow(1)$ : If $\left(T_{\mathbf{W}, \mathbf{w}}^{*} T_{\mathbf{V}, \mathbf{v}} Q\right)^{2}=T_{\mathbf{W}, \mathbf{w}}^{*} T_{\mathbf{V}, \mathbf{v}} Q$ then $K_{\mathcal{W}}=\operatorname{Ker}\left(T_{\mathbf{W}, \mathbf{w}}^{*} T_{\mathbf{V}, \mathbf{v}} Q\right) \oplus \operatorname{Im}\left(T_{\mathbf{W}, \mathbf{w}}^{*} T_{\mathbf{V}, \mathbf{v}} Q\right)$. Since $T_{\mathbf{W}, \mathbf{w}}^{*} \mid \mathcal{V}$ is injective we have $\operatorname{Ker}\left(T_{\mathbf{W}, \mathbf{w}}^{*} T_{\mathbf{V}, \mathbf{v}} Q\right)=\operatorname{Ker}\left(T_{\mathbf{V}, \mathbf{v}} Q\right)$ and so $K_{\mathcal{W}}=\operatorname{Ker}\left(T_{\mathbf{V}, \mathbf{v}} Q\right) \oplus$ $\operatorname{Im}\left(T_{\mathbf{W}, \mathbf{w}}^{*} T_{\mathbf{V}, \mathbf{v}} Q\right)$. Therefore, since $\operatorname{Im}\left(T_{\mathbf{V}, \mathbf{v}} Q\right)=\mathcal{V}$,

$$
\mathcal{V}=\left\{T_{\mathbf{V}, \mathbf{v}} Q\left\{f_{i}\right\}_{i \in I}:\left\{f_{i}\right\}_{i \in I} \in \operatorname{Im}\left(T_{\mathbf{W}, \mathbf{w}}^{*} T_{\mathbf{V}, \mathbf{v}} Q\right)\right\} .
$$

Let now $f \in \mathcal{V}$ with $f=T_{\mathbf{V}, \mathbf{v}} Q\left\{f_{i}\right\}_{i \in I}$ for some $\left\{f_{i}\right\}_{i \in I} \in \operatorname{Im}\left(T_{\mathbf{W}, \mathbf{w}}^{*} T_{\mathbf{V}, \mathbf{v}} Q\right)$. Then 


$$
T_{\mathbf{V}, \mathbf{v}} Q T_{\mathbf{W}, \mathbf{w}}^{*} f=T_{\mathbf{V}, \mathbf{v}} Q T_{\mathbf{W}, \mathbf{w}}^{*} T_{\mathbf{V}, \mathbf{v}} Q\left\{f_{i}\right\}_{i \in I}=T_{\mathbf{V}, \mathbf{v}} Q\left\{f_{i}\right\}_{i \in I}=f .
$$

In a similar way it can be proved that $(3) \Leftrightarrow(8)$.

If (1) is satisfied then $T_{\mathbf{V}, \mathbf{v}}$ is onto and hence $(\mathbf{V}, \mathbf{v})$ is a fusion frame for $\mathcal{V}$. Similarly $(\mathbf{W}, \mathbf{w})$ is a a fusion frame for $\mathcal{W}$.

We will now present two special types of linear transformations $Q$ that make the reconstruction formula that follows from (3.1) simpler. In order to do that we need the selfadjoint operator $M_{J, \mathbf{W}}: K_{\mathcal{W}} \rightarrow K_{\mathcal{W}}, M_{J, \mathbf{W}}\left(f_{i}\right)_{i \in I}=\left(\chi_{J}(i) f_{i}\right)_{i \in I}$. We just write $M_{J}$ if it clear to which $\mathbf{W}$ we refer to. We use the notation $M_{\{j\}, \mathbf{W}}=M_{j, \mathbf{W}}$ and $M_{\{j\}}=M_{j}$.

Definition 3.5. Let $Q \in L\left(K_{\mathcal{W}}, K_{\mathcal{V}}\right)$.

(1) If $Q M_{j, \mathbf{W}} K_{\mathcal{W}} \subseteq M_{j, \mathbf{V}} K_{\mathcal{V}}$ for each $j \in I, Q$ is called block-diagonal.

(2) If $Q M_{j, \mathbf{W}} K_{\mathcal{W}}=M_{j, \mathbf{V}} K_{\mathcal{V}}$ for each $j \in I, Q$ is called component preserving.

Note that $Q$ is block-diagonal if and only if $Q M_{J, \mathbf{W}}=M_{J, \mathbf{V}} Q$ for each $J \subseteq I$, or equivalently, $Q M_{j, \mathbf{W}}=M_{j, \mathbf{V}} Q$ for each $j \in I$. Observe that if $Q$ is block-diagonal, then $Q^{*}$ is block-diagonal. If in Definition $3.2 Q$ is block-diagonal (component preserving) we say that $(\mathbf{V}, \mathbf{v})$ is a block-diagonal dual fusion frame (component preserving dual fusion frame) of $(\mathbf{W}, \mathbf{w})$.

Another motivation for introducing the notion of oblique duality as in Definition 3.2 is to obtain flexibility, therefore asking for restrictions only when needed. The general framework provided by Definition 3.2 allows to adjust to the problem at hand. This is another reason to start with the most general class and then naturally arise the particular classes with which we work here: block-diagonal and component preserving oblique dual fusion frames. As we will see in Lemma 6.2, $Q$ is component preserving for oblique dual fusion frames obtained from the oblique left inverses of $T_{\mathbf{W}, \mathbf{w}}^{*}$. Also, $Q$ is block-diagonal for oblique dual fusion frame systems (see Definition 3.6 and Remark 3.7).

If $Q$ is block-diagonal, from (3.1) we obtain the following reconstruction formula:

$$
\pi_{\mathcal{V}, \mathcal{W} \perp} f=\sum_{j \in I} v_{j} w_{j} Q_{j} f, \forall f \in \mathcal{H}
$$

where $Q_{j}: \mathcal{H} \rightarrow V_{j}$ is given by $Q_{j} f:=\left(Q M_{j}\left(\pi_{W_{i}} f\right)_{i \in I}\right)_{j}$. For each $j \in I, Q_{j}$ is a bounded linear operator. Observe that $W_{j}^{\perp} \subseteq \operatorname{ker}\left(Q_{j}\right)$ and we can recover the block-diagonal (or component preserving) mapping $Q$ as $Q=\oplus_{j \in I} Q_{j}$. 
Note that $(\mathbf{V}, \mathbf{v})$ is a $Q$-oblique dual fusion frame of $(\mathbf{W}, \mathbf{w})$ if and only if $(\mathbf{V}, \mathbf{c v})$ is a $\frac{1}{\mathbf{c}} Q$-oblique dual fusion frame of $(\mathbf{W}, \mathbf{w})$, where $\mathbf{c v}=\left\{c_{i} w_{i}\right\}_{i \in I}$ and $\frac{1}{\mathbf{c}} Q=\oplus_{j \in I}\left(\frac{1}{c_{j}} Q_{j}\right)$, with $0<\inf _{i \in I} c_{i} \leq$ $\sup _{i \in I} c_{i}<\infty$. Both oblique dual fusion frames lead to the same reconstruction formula. This freedom for the weights is desirable because we can select those $\mathbf{v}$ such that the pair $(\mathbf{V}, \mathbf{v})$ is the most suitable to treat simultaneously another problem not related with the reconstruction formula.

We observe that in each term of (3.2) we can think the product $v_{j} w_{j}\left\|Q_{j}\right\|$ as the weight that accompanies the pair of subspaces $W_{j}$ and $V_{j}$, determining their importance in the reconstruction.

3.3. Oblique dual fusion frame systems. We will define and study in this section the concept of oblique dual fusion frame systems. In order to do that, we will need the following operator, which we introduced in [12], and which establishes the connection between the synthesis operator of a fusion frame system and the synthesis operator of its associated frame.

Let $(\mathbf{W}, \mathbf{w})$ be a Bessel fusion sequence for $\mathcal{W}$ and $\mathcal{F}_{i}$ be a frame for $W_{i}$ with frame bounds $\alpha_{i}, \beta_{i}$ for each $i \in I$ such that $\sup _{i \in I} \beta_{i}=\beta<\infty$. Let

$$
C_{\mathcal{F}}: \oplus_{i \in I} \ell^{2}\left(L_{i}\right) \rightarrow K_{\mathcal{W}}, C_{\mathcal{F}}\left(\left(x_{i, l}\right)_{l \in L_{i}}\right)_{i \in I}=\left(T_{\mathcal{F}_{i}}\left(x_{i, l}\right)_{l \in L_{i}}\right)_{i \in I} .
$$

Note that $C_{\mathcal{F}}$ is a surjective bounded operator with $\left\|C_{\mathcal{F}}\right\| \leq \beta$. Its adjoint is $C_{\mathcal{F}}^{*}: K_{\mathcal{W}} \rightarrow$ $\oplus_{i \in I} \ell^{2}\left(L_{i}\right)$, given by $C_{\mathcal{F}}^{*}\left(g_{i}\right)_{i \in I}=\left(T_{\mathcal{F}_{i}}^{*} g_{i}\right)_{i \in I}$ and satisfies $\left\|C_{\mathcal{F}}^{*}\left(g_{i}\right)_{i \in I}\right\| \leq \beta\left\|\left(g_{i}\right)_{i \in I}\right\|$. If $0<$ $\alpha=\inf _{i \in I} \alpha_{i}$, we also have $\alpha\left\|\left(g_{i}\right)_{i \in I}\right\| \leq\left\|C_{\mathcal{F}}^{*}\left(g_{i}\right)_{i \in I}\right\|$. The bounded left inverses of $C_{\mathcal{F}}^{*}$ are all $C_{\widetilde{\mathcal{F}}} \in L\left(\oplus_{i \in I} \ell^{2}\left(L_{i}\right), K_{\mathcal{W}}\right)$ such that $\widetilde{\mathcal{F}}_{i}$ is a dual frame of $\mathcal{F}_{i}$ with upper frame bound $\widetilde{\beta}_{i}$ for each $i \in I$ such that $\sup _{i \in I} \widetilde{\beta}_{i}<\infty$. Observe that

$$
T_{\mathbf{w} \mathcal{F}}=T_{\mathbf{W}, \mathbf{w}} C_{\mathcal{F}} \text { and } T_{\mathbf{W}, \mathbf{w}}=T_{\mathbf{w} \mathcal{F}} C_{\widetilde{\mathcal{F}}}^{*} .
$$

We define oblique dual fusion frame systems as follows:

Definition 3.6. Let $\mathcal{W}$ and $\mathcal{V}$ be two closed subspaces of $\mathcal{H}$ such that $\mathcal{H}=\mathcal{V} \oplus \mathcal{W}^{\perp}$. Let $(\mathbf{W}, \mathbf{w}, \mathcal{F})$ be a fusion frame system for $\mathcal{W}$ with upper local frame bound $\beta_{i}$ for each $i \in I$ such that $\sup _{i \in I} \beta_{i}<\infty,(\mathbf{V}, \mathbf{v}, \mathcal{G})$ be a fusion frame system for $\mathcal{V}$ with local upper frame bound $\widetilde{\beta}_{i}$ for each $i \in I$ such that $\sup _{i \in I} \widetilde{\beta}_{i}<\infty$ and $\left|\mathcal{F}_{i}\right|=\left|\mathcal{G}_{i}\right|$ for each $i \in I$. Then $(\mathbf{V}, \mathbf{v}, \mathcal{G})$ is an oblique dual fusion frame system of $(\mathbf{W}, \mathbf{w}, \mathcal{F})$ on $\mathcal{V}$ if $(\mathbf{V}, \mathbf{v})$ is a $C_{\mathcal{G}} C_{\mathcal{F}}^{*}$-oblique dual fusion frame of $(\mathbf{W}, \mathbf{w})$ on $\mathcal{V}$.

Remark 3.7. It is easy to see that the operator $C_{\mathcal{G}} C_{\mathcal{F}}^{*}: K_{\mathcal{W}} \rightarrow K_{\mathcal{V}}, C_{\mathcal{G}} C_{\mathcal{F}}^{*}\left(f_{i}\right)_{i \in I}=\left(T_{\mathcal{G}_{i}} T_{\mathcal{F}_{i}}^{*} f_{i}\right)_{i \in I}$ is block-diagonal. 
If $C_{\mathcal{G}} C_{\mathcal{F}}^{*}$ in Definition 3.6 is component preserving, we call $(\mathbf{V}, \mathbf{v}, \mathcal{G})$ a component preserving oblique dual fusion frame system of $(\mathbf{W}, \mathbf{w}, \mathcal{F})$.

Remark 3.8. If $\mathcal{W}=\mathcal{V}$ we have in Definition 3.2 and in Definition 3.6 the concepts of dual fusion frame and dual fusion frame system, respectively, considered in [11, 12. In this case, we simply say that $(\mathbf{V}, \mathbf{v})$ is a $Q$ - dual fusion frame of $(\mathbf{W}, \mathbf{w})$ or that $(\mathbf{V}, \mathbf{v}, \mathcal{G})$ is a dual fusion frame system of $(\mathbf{W}, \mathbf{w}, \mathcal{F})$.

\section{Relation between block-diagonal oblique dual frames, oblique Dual fusion FRAME SYSTEMS AND OBLIQUE DUAL FRAMES}

We defined oblique dual fusion frame systems in terms of (block-diagonal) oblique dual fusion frames (see Definition 3.6 and Remark 3.7). Conversely, we can always associate to a block-diagonal oblique dual fusion frame pair an oblique dual fusion frame system pair. In order to see this we need the following two auxiliary results.

Lemma 4.1. If $A \in L(\mathcal{H}, \mathcal{K})$, then there exists a frame $\mathcal{F}$ for $\mathcal{H}$ and a frame $\mathcal{G}$ for $\mathcal{K}$ such that $|\mathcal{F}|=|\mathcal{G}|$ and $A=T_{\mathcal{G}} T_{\mathcal{F}}^{*}$. We can choose $\mathcal{F}$ and $\mathcal{G}$ in such a way that their frame bounds are 1,2 and $1,1+\|A\|^{2}$, respectively.

Proof. Let $\mathcal{F}$ be any frame for $\mathcal{H}$ and $\widetilde{\mathcal{F}}$ be any dual frame of $\mathcal{F}$. Then $A \widetilde{\mathcal{F}}$ is a frame for $\operatorname{Im}(A)$, $|A \widetilde{\mathcal{F}}|=|\mathcal{F}|$ and $A=T_{A \widetilde{\mathcal{F}}} T_{\mathcal{F}}^{*}$.

If $\operatorname{Im}(A) \neq \mathcal{K}$ let $\mathcal{G}=\left\{g_{i}\right\}_{i \in J}$ be any frame for $\operatorname{Im}(A)^{\perp}$. If $\widetilde{\mathcal{G}}$ is the family indexed by $J$ with all its elements equal to the zero of $\mathcal{H}$, then $T_{\mathcal{G}} T_{\widetilde{\mathcal{G}}}^{*}=0$. We can also construct $\widetilde{\mathcal{G}}$ with not all of its elements equal to zero that has this property. For this, we consider a frame $\mathcal{G}=\left\{g_{j}\right\}_{j \in J}$ for $\operatorname{Im}(A)^{\perp}$ that is not a basis. Let $\left\{c_{m}\right\}_{m \in \mathbb{M}}$ be an orthonormal basis for $\operatorname{Ker}\left(T_{\mathcal{G}}\right) \subset \ell^{2}(J)$ where $\mathbb{M}=\mathbb{N}$ or $\mathbb{M}=\{1, \ldots, M\}$. Let $\left\{e_{l}\right\}_{l \in \mathbb{L}}$ be an orthonormal basis for $\mathcal{H}$ where $\mathbb{L}=\mathbb{N}$ or $\mathbb{L}=\{1, \ldots, L\}$. Let $\mathbb{I}$ any finite subset of $\mathbb{M} \cap \mathbb{L}$ and $\widetilde{g}_{j}=\sum_{l \in \mathbb{I}} \overline{c_{l}(j)} e_{l}$ for each $j \in J$. By the linear independence of $\left\{e_{l}\right\}_{l \in \mathbb{L}}$ and $\left\{c_{m}\right\}_{m \in \mathbb{M}}$, the vectors $\widetilde{g}_{j}$ can not be all equal to 0 . We have $\sum_{j \in J}\left|\left\langle f, \widetilde{g}_{j}\right\rangle\right|^{2} \leq \sum_{l \in \mathbb{I}}\left|\left\langle f, e_{l}\right\rangle\right|^{2} \sum_{j \in J}\left|c_{l}(j)\right|^{2} \leq\|f\|^{2}$. Therefore, $\left\{\widetilde{g}_{j}\right\}_{j \in J}$ is a Bessel sequence with Bessel bound 1. Note that if $d \in \ell^{2}(J)$ and $f \in \mathcal{H}$, then $\left\langle T_{\widetilde{\mathcal{G}}} d, f\right\rangle=\left\langle\sum_{j \in J} d(j) \sum_{l \in \mathbb{I}} \overline{c_{l}(j)} e_{l}, f\right\rangle=$ $\sum_{j \in J} d(j) \sum_{l \in \mathbb{I}} \overline{c_{l}(j)}\left\langle e_{l}, f\right\rangle=\left\langle d, \sum_{l \in \mathbb{I}}\left\langle f, e_{l}\right\rangle c_{l}\right\rangle$, and then $T_{\mathcal{G}} T_{\widetilde{\mathcal{G}}}^{*} f=\sum_{l \in \mathbb{I}}\left\langle f, e_{l}\right\rangle T_{\mathcal{G}} c_{l}=0$ since $c_{l} \in$ $\operatorname{Ker}\left(T_{\mathcal{G}}\right)$ for each $l \in \mathbb{I}$. 
Finally, $\{\mathcal{F}, \widetilde{\mathcal{G}}\}$ is a frame for $\mathcal{H},\{A \widetilde{\mathcal{F}}, \mathcal{G}\}$ is a frame for $\mathcal{K},|\{\mathcal{F}, \widetilde{\mathcal{G}}\}|=|\{A \widetilde{\mathcal{F}}, \mathcal{G}\}|$ and $A=$ $T_{\{A \widetilde{\mathcal{F}}, \mathcal{G}\}} T_{\{\mathcal{F}, \widetilde{\mathcal{G}}\}}^{*}$.

If we choose $\mathcal{F}, \widetilde{\mathcal{F}}$ and $\mathcal{G}$ to be Parseval, the frame bounds of $\{\mathcal{F}, \widetilde{\mathcal{G}}\}$ and $\{A \widetilde{\mathcal{F}}, \mathcal{G}\}$ are 1,2 and $1,1+\|A\|^{2}$, respectively.

Corollary 4.2. If $Q \in L\left(K_{\mathcal{W}}, K_{\mathcal{V}}\right)$ is block-diagonal then there exists a frame $\mathcal{F}_{i}$ for $W_{i}$ with frame bounds $\alpha_{i}, \beta_{i}$ for each $i \in I$, satisfying $0<\inf _{i \in I} \alpha_{i} \leq \sup _{i \in I} \beta_{i}<\infty$, and a frame $\mathcal{G}_{i}$ for $V_{i}$ with $\left|\mathcal{F}_{i}\right|=\left|\mathcal{G}_{i}\right|$ and frame bounds $\widetilde{\alpha}_{i}, \widetilde{\beta}_{i}$ for each $i \in I$, satisfying $0<\inf _{i \in I} \widetilde{\alpha}_{i} \leq \sup _{i \in I} \widetilde{\beta}_{i}<\infty$ such that $Q=C_{\mathcal{G}} C_{\mathcal{F}}^{*}$.

Proof. By Lemma 4.1, for each $i \in I$ there exists frames $\mathcal{F}_{i}$ for $W_{i}$ with frame bounds $\alpha_{i}=1$, $\beta_{i}=2$ and $\mathcal{G}_{i}$ for $V_{i}$ with frame bounds $\widetilde{\alpha}_{i}=1, \widetilde{\beta}_{i}=1+\left\|Q_{i}\right\|^{2}$ such that $Q_{i}=T_{\mathcal{G}_{i}} T_{\mathcal{F}_{i}}^{*}$. Thus, $Q=C_{\mathcal{G}} C_{\mathcal{F}}^{*}$. Moreover, $\left\|Q_{i}\right\| \leq\|Q\|$ for each $i \in I$.

The next theorem asserts that a block-diagonal oblique dual fusion frame pair can always be viewed as an oblique dual fusion frame system pair.

Theorem 4.3. Let $\mathcal{W}$ and $\mathcal{V}$ be two closed subspaces of $\mathcal{H}$. Let $(\mathbf{W}, \mathbf{w})$ be a fusion frame for $\mathcal{W}$ and let $(\mathbf{V}, \mathbf{v})$ be a block diagonal $Q$-oblique dual fusion frame of $(\mathbf{W}, \mathbf{w})$ on $\mathcal{V}$. Then there exists a frame $\mathcal{F}_{i}$ for $W_{i}$ with frame bounds $\alpha_{i}, \beta_{i}$ for each $i \in I$ such that $0<\inf _{i \in I} \alpha_{i} \leq \sup _{i \in I} \beta_{i}<\infty$ and a frame $\mathcal{G}_{i}$ for $V_{i}$ with frame bounds $\widetilde{\alpha}_{i}, \widetilde{\beta}_{i}$ for each $i \in I$ such that $0<\inf _{i \in I} \widetilde{\alpha}_{i} \leq \sup _{i \in I} \widetilde{\beta}_{i}<\infty$, such that $(\mathbf{V}, \mathbf{v}, \mathcal{G})$ is a dual fusion frame system of $(\mathbf{W}, \mathbf{w}, \mathcal{F})$ and $Q=C_{\mathcal{G}} C_{\mathcal{F}}^{*}$.

Proof. It is a consequence of Definition 3.2 . Corollary 4.2 and Definition 3.6 .

The following theorem establishes the connection between the notions of oblique dual fusion frame system and oblique dual frame.

Theorem 4.4. Let $\mathcal{W}$ and $\mathcal{V}$ be two closed subspaces of $\mathcal{H}$ such that $\mathcal{H}=\mathcal{V} \oplus \mathcal{W}^{\perp}$. Let $(\mathbf{W}, \mathbf{w}, \mathcal{F})$ be a Bessel fusion system for $\mathcal{W}$ such that $\mathcal{F}_{i}$ has upper frame bound $\beta_{i}$ for each $i \in I$ with $\sup _{i \in I} \beta_{i}<\infty$, and let $(\mathbf{V}, \mathbf{v}, \mathcal{G})$ be a Bessel fusion system for $\mathcal{V}$ such that $\mathcal{G}_{i}$ has upper frame bound $\widetilde{\beta}_{i}$ for each $i \in I$ with $\sup _{i \in I} \widetilde{\beta}_{i}<\infty$. If $\left|\mathcal{F}_{i}\right|=\left|\mathcal{G}_{i}\right|$ for each $i \in I$ then the following conditions are equivalent:

(1) $\mathbf{w} \mathcal{F}$ and $\mathbf{v} \mathcal{G}$ are oblique dual frames for $\mathcal{H}$. 
$(2)(\mathbf{V}, \mathbf{v}, \mathcal{G})$ is an oblique dual fusion frame system of $(\mathbf{W}, \mathbf{w}, \mathcal{F})$ on $\mathcal{V}$.

Proof. It follows from Theorem 2.9, the equality $T_{\mathbf{v} \mathcal{G}} T_{\mathbf{w} \mathcal{F}}^{*}=T_{\mathbf{V}, \mathbf{v}} C_{\mathcal{G}} C_{\mathcal{F}}^{*} T_{\mathbf{W}, \mathbf{w}}^{*}$, 4, Lemma 6.3.2] and Lemma 3.4

\section{Duals and oblique duals}

Oblique duality is a generalization of duality. The results in this section provide methods to obtain dual fusion frames from oblique dual fusion frmaes and viceversa.

Proposition 5.1. Let $\mathcal{W}$ and $\mathcal{V}$ be two closed subspaces of $\mathcal{H}$ such that $\mathcal{H}=\mathcal{V} \oplus \mathcal{W}^{\perp}$. Let $(\mathbf{W}, \mathbf{w}, \mathcal{F})$ be a fusion frame system for $\mathcal{W}$ with local upper frame bounds $\beta_{i}$ for each $i \in I$ such that $\sup _{i \in I} \beta_{i}<\infty,(\mathbf{V}, \mathbf{v}, \mathcal{G})$ be a fusion frame system for $\mathcal{V}$ with local upper frame bounds $\widetilde{\beta}_{i}$ for each $i \in I$ such that $\sup _{i \in I} \widetilde{\beta}_{i}<\infty$ and $\left|\mathcal{F}_{i}\right|=\left|\mathcal{G}_{i}\right|$ for each $i \in I$. If $(\mathbf{V}, \mathbf{v}, \mathcal{G})$ is an oblique dual fusion frame system of $(\mathbf{W}, \mathbf{w}, \mathcal{F})$ on $\mathcal{V}$, then $\left(\pi_{\mathcal{W}}(\mathbf{V}), \mathbf{v}, \pi_{\mathcal{W}}(\mathcal{G})\right)$ is a dual fusion frame system of $(\mathbf{W}, \mathbf{w}, \mathcal{F})$ for $\mathcal{W}$ and $\left(\pi_{\mathcal{V}}(\mathbf{W}), \mathbf{w}, \pi_{\mathcal{V}}(\mathcal{F})\right)$ is a dual fusion frame system of $(\mathbf{V}, \mathbf{v}, \mathcal{G})$ for $\mathcal{V}$.

Proof. Since $\mathcal{V} \cap \mathcal{W}^{\perp}=\{0\}$, if $f \in \mathcal{V}$ is such that $\pi_{\mathcal{W}} f=0$, then $f=0$. So, $\pi_{\mathcal{W}}(\mathcal{V}) \neq\{0\}$. By [4, Proposition 5.3.1], $\pi_{\mathcal{W}}\left(\mathcal{G}_{i}\right)$ is a frame for $\pi_{\mathcal{W}}\left(V_{i}\right)$ with upper frame bound $\widetilde{\beta}_{i}$ for each $i \in I$ and $\pi_{\mathcal{W}}(\mathcal{G})$ is a frame for $\pi_{\mathcal{W}}(\mathcal{V})$. By Theorem 2.9] $\left(\pi_{\mathcal{W}}(\mathbf{V}), \mathbf{v}, \pi_{\mathcal{W}}(\mathcal{G})\right)$ is a Bessel fusion sequence for $\pi_{\mathcal{W}}(\mathcal{V})$. We have $T_{\mathbf{V}, \mathbf{v}} C_{\mathcal{G}} C_{\mathcal{F}}^{*} T_{\mathbf{W}, \mathbf{w}}^{*}=\pi_{\mathcal{V}, \mathcal{W}}$. Also $T_{\pi_{\mathcal{W}}(\mathbf{V}), \mathbf{v}} C_{\pi_{\mathcal{W}}(\mathcal{G})}=\pi_{\mathcal{W}} T_{\mathbf{v} \mathcal{G}}$. Hence,

$$
T_{\pi_{\mathcal{W}}(\mathbf{V}), \mathbf{v}} C_{\pi_{\mathcal{W}}(\mathcal{G})} C_{\mathcal{F}}^{*} T_{\mathbf{W}, \mathbf{w}}^{*}=\pi_{\mathcal{W}} T_{\mathbf{v} \mathcal{G}} C_{\mathcal{F}}^{*} T_{\mathbf{W}, \mathbf{w}}^{*}=\pi_{\mathcal{W}} T_{\mathbf{V}, \mathbf{v}} C_{\mathcal{G}} C_{\mathcal{F}}^{*} T_{\mathbf{W}, \mathbf{w}}^{*}=\pi_{\mathcal{W}} \pi_{\mathcal{V}, \mathcal{W} \perp}=\pi_{\mathcal{W}}
$$

By [11, Lemma 3.2], $\left(\pi_{\mathcal{W}}(\mathbf{V}), \mathbf{v}, \pi_{\mathcal{W}}(\mathcal{G})\right)$ is a dual fusion frame system of $(\mathbf{W}, \mathbf{w}, \mathcal{F})$ for $\mathcal{W}$.

The other assertion is proved in a similar way.

By Theorem 4.3 and Definition 3.6 we obtain the following Corollary:

Corollary 5.2. Let $\mathcal{W}$ and $\mathcal{V}$ be two closed subspaces of $\mathcal{H}$ such that $\mathcal{H}=\mathcal{V} \oplus \mathcal{W}^{\perp}$. If $(\mathbf{V}, \mathbf{v})$ is a block-diagonal $\oplus_{i \in I} Q_{i}$-oblique dual fusion frame of $(\mathbf{W}, \mathbf{w})$ on $\mathcal{V}$, then $\left(\pi_{\mathcal{W}}(\mathbf{V})\right.$, $\left.\mathbf{v}\right)$ is a $\oplus_{i \in I} \pi_{\mathcal{W}} Q_{i^{-}}$ block-diagonal dual fusion frame of $(\mathbf{W}, \mathbf{w})$ for $\mathcal{W}$ and $\left(\pi_{\mathcal{V}}(\mathbf{W}), \mathbf{w}\right)$ is a $\oplus_{i \in I} \pi_{\mathcal{V}} Q_{i}$-block-diagonal dual fusion frame of $(\mathbf{V}, \mathbf{v})$ for $\mathcal{V}$.

The following two results can be proved in a similar way as Proposition 5.1 and Corollary 5.2, respectively. 
Proposition 5.3. Let $\mathcal{W}$ and $\mathcal{V}$ be two closed subspaces of $\mathcal{H}$ such that $\mathcal{H}=\mathcal{V} \oplus \mathcal{W}^{\perp}$. Let $(\mathbf{W}, \mathbf{w}, \mathcal{F})$ be a fusion frame system for $\mathcal{W}$ with local upper frame bound $\beta_{i}$ for each $i \in I$ such that $\sup _{i \in I} \beta_{i}<\infty,(\widetilde{\mathbf{W}}, \widetilde{\mathbf{w}}, \widetilde{\mathcal{F}})$ be a fusion frame system for $\mathcal{W}$ with local upper frame bound $\widetilde{\beta}_{i}$ for each $i \in I$ such that $\sup _{i \in I} \widetilde{\beta}_{i}<\infty$ and $\left|\mathcal{F}_{i}\right|=\left|\widetilde{\mathcal{F}}_{i}\right|$ for each $i \in I$. If $(\widetilde{\mathbf{W}}, \widetilde{\mathbf{w}}, \widetilde{\mathcal{F}})$ is a dual fusion frame system of $(\mathbf{W}, \mathbf{w}, \mathcal{F})$, then $\left(\pi_{\mathcal{V}, \mathcal{W}^{\perp}} \widetilde{\mathbf{W}}, \widetilde{\mathbf{w}}, \pi_{\mathcal{V}, \mathcal{W}^{\perp}} \widetilde{\mathcal{F}}\right)$ is an oblique dual fusion frame system of $(\mathbf{W}, \mathbf{w}, \mathcal{F})$ on $\mathcal{V}$.

Corollary 5.4. Let $\mathcal{W}$ and $\mathcal{V}$ be two closed subspaces of $\mathcal{H}$ such that $\mathcal{H}=\mathcal{V} \oplus \mathcal{W}^{\perp}$. If $(\widetilde{\mathbf{W}}, \widetilde{\mathbf{w}})$ is a block-diagonal $\oplus_{i \in I} Q_{i}$-dual fusion frame of $(\mathbf{W}, \mathbf{w})$ for $\mathcal{W}$, then $\left(\pi_{\mathcal{V}, \mathcal{W} \perp}(\widetilde{\mathbf{W}}), \widetilde{\mathbf{w}}\right)$ is a block-diagonal $\oplus_{i \in I} \pi_{\mathcal{V}, \mathcal{W}^{\perp}} Q_{i}$-oblique dual fusion frame of $(\mathbf{W}, \mathbf{w})$ on $\mathcal{V}$

\section{OBlique dual FAMILIES}

In [11, 12, it is shown that component preserving dual fusion frames are related to the left inverses of the analysis operator. In this section we will show that analogous results are valid for component preserving oblique dual fusion frames.

The following Lemma can be deduced from Corollary 5.2, [11, Lemma 3.4], Lemma 2.1 and Proposition 2.3. Nevertheless, we include a short direct proof of it.

Lemma 6.1. Let $\mathcal{W}$ and $\mathcal{V}$ be two closed subspaces of $\mathcal{H}$ such that $\mathcal{H}=\mathcal{V} \oplus \mathcal{W}^{\perp}$. Let $(\mathbf{W}$, w) be a fusion frame for $\mathcal{W}$. If $(\mathbf{V}, \mathbf{v})$ is a $Q$-component preserving oblique dual fusion frame of $(\mathbf{W}, \mathbf{w})$ on $\mathcal{V}$ then $V_{i}=A M_{i} K_{\mathcal{W}}$ for each $i \in I$ for $A=T_{\mathbf{V}, \mathbf{v}} Q \in \mathfrak{L}_{T_{\mathbf{W}, \mathbf{w}}^{*}}^{\mathcal{V}} \mathcal{W}^{\perp}$.

Proof. Let $Q \in L\left(\mathcal{K}_{\mathcal{W}}, \mathcal{K}_{\mathcal{V}}\right)$ be component preserving such that $T_{\mathbf{V}, \mathbf{v}} Q T_{\mathbf{W}, \mathbf{w}}^{*}=\pi_{\mathcal{V}, \mathcal{W} \perp}$ and let $A=$ $T_{\mathbf{V}, \mathbf{v}} Q$. Clearly, $A \in \mathfrak{L}_{T_{\mathbf{W}, \mathbf{w}}^{*}, \mathcal{W}^{\perp}}$. Using that $Q$ is component preserving, $A M_{i} K_{\mathcal{W}}=T_{\mathbf{V}, \mathbf{v}} Q M_{i} K_{\mathcal{W}}=$ $V_{i}$ for each $i \in I$.

A reciprocal of the previous result is:

Lemma 6.2. Let $\mathcal{W}$ and $\mathcal{V}$ be two closed subspaces of $\mathcal{H}$ such that $\mathcal{H}=\mathcal{V} \oplus \mathcal{W}^{\perp}$. Let $(\mathbf{W}$, w) be a fusion frame for $\mathcal{W}, A \in \mathfrak{L}_{T_{\mathbf{W}, \mathbf{w}}^{*}, \mathcal{W}^{\perp}}$ and $V_{i}=A M_{i} \mathcal{K}_{\mathcal{W}}$ for each $i \in I$. If $(\mathbf{V}, \mathbf{v})$ is a Bessel fusion sequence for $\mathcal{V}$ and

$$
Q_{A, \mathbf{v}}: K_{\mathcal{W}} \rightarrow K_{\mathcal{V}}, Q_{A, \mathbf{v}}\left(f_{j}\right)_{j \in I}=\left(\frac{1}{v_{i}} A M_{i}\left(f_{j}\right)_{j \in I}\right)_{i \in I}
$$


is a well defined bounded operator, then $(\mathbf{V}, \mathbf{v})$ is a $Q_{A, \mathbf{v}}$-component preserving oblique dual fusion frame of $(\mathbf{W}, \mathbf{w})$ on $\mathcal{V}$.

Proof. From the hypotheses, $\overline{\operatorname{span}} \bigcup_{i \in I} V_{i}=\operatorname{Im}(A)=\mathcal{V}, Q_{A, \mathbf{v}}$ is component preserving and $A=$ $T_{\mathbf{V}, \mathbf{v}} Q_{A, \mathbf{v}}$. Since $A \in \mathfrak{L}_{T_{\mathbf{W}, \mathbf{w}}^{*}, \mathcal{W}^{\perp}}^{*}, T_{\mathbf{V}, \mathbf{v}} Q_{A, \mathbf{v}} T_{\mathbf{W}, \mathbf{w}}^{*}=\pi_{\mathcal{V}, \mathcal{W}^{\perp}}$. So $(\mathbf{V}, \mathbf{v})$ is a $Q_{A, \mathbf{v}^{-c o m p o n e n t ~ p r e s e r v i n g ~}}$ oblique dual fusion frame of $(\mathbf{W}, \mathbf{w})$ on $\mathcal{V}$.

See [11, Remark 3.6] for sufficient conditions for $(\mathcal{V}, v)$ being a Bessel fusion sequence for $\mathcal{V}$ and for $Q_{A, v}$ being a well defined bounded operator in Lemma 6.2 For the case in which $\mathcal{W}$ and $\mathcal{V}$ are finite-dimensional, Lemma 6.1 and Lemma 6.2 lead to the following characterization:

Theorem 6.3. Let $\mathcal{W}$ and $\mathcal{V}$ be finite-dimensional subspaces of the Hilbert space $\mathcal{H}$ such that $\mathcal{H}=\mathcal{V} \oplus \mathcal{W}^{\perp}$. Let $\mathcal{W}$ and $\mathcal{V}$ be two subspaces of $\mathcal{H}$ such that $\mathcal{H}=\mathcal{V} \oplus \mathcal{W}^{\perp}$. Let $(\mathbf{W}, \mathbf{w})$ be a fusion frame for $\mathcal{W}$. Then $(\mathbf{V}, \mathbf{v})$ is a $Q$-component preserving oblique dual fusion frame of $(\mathbf{W}, \mathbf{w})$ on $\mathcal{V}$ if and only if $V_{i}=A M_{i} K_{\mathcal{W}}$ for each $i \in I$ and $Q=Q_{A, \mathbf{v}}$, for some $A \in \mathfrak{L}_{T_{\mathbf{W}, \mathbf{w}}^{\mathcal{V}}, \mathcal{W}^{\perp}}$ with $\operatorname{Im}(A)=\mathcal{V}$. Moreover, any element of $\mathfrak{L}_{T_{\mathbf{W}, \mathbf{w}}^{*}, \mathcal{W}^{\perp}}^{*}$ with $\operatorname{Im}(A)=\mathcal{V}$ is of the form $T_{\mathbf{V}, \mathbf{v}} Q$ where $(\mathbf{V}, \mathbf{v})$ is some $Q$-component preserving oblique dual fusion frame of $(\mathbf{W}, \mathbf{w})$ on $\mathcal{V}$.

The above results show that component preserving oblique dual fusion frames can be obtained in a similar manner as in the vectorial case (see [4, Lemma 6.3.5]).

Remark 6.4. By Proposition [2.3. Theorem 6.3 and [12, Theorem 3.5], if $\mathcal{W}$ and $\mathcal{V}$ are finitedimensional, there is a bijection between component preserving dual and component preserving oblique dual fusion frames.

Remark 6.5. As a consequence of Theorem 6.3 , if $\mathcal{W}$ and $\mathcal{V}$ are finite-dimensional we can always associate to any $Q$-oblique dual fusion frame $(\mathbf{V}, \mathbf{v})$ of $(\mathbf{W}, \mathbf{w})$ the $Q_{A, \tilde{\mathbf{v}}}$-component preserving oblique dual fusion frame $\left\{\left(A M_{i} K_{\mathcal{W}}, \tilde{v}_{i}\right)\right\}_{i \in I}$ with $A=T_{\mathbf{V}, \mathbf{v}} Q$ and $\left\{\widetilde{v}_{i}\right\}_{i \in I}$ arbitrary weights. Furthermore, if $Q$ is block-diagonal, then $Q_{T_{\mathbf{v}, \mathbf{v}} Q, \mathbf{v}}\left(f_{i}\right)_{i \in I}=Q\left(f_{i}\right)_{i \in I}$ for each $\left(f_{i}\right)_{i \in I} \in K_{\mathcal{W}}$.

Remark 6.6. It is easy to see that

$$
\begin{aligned}
\mathfrak{L}_{T_{\mathbf{W}, \mathbf{w}}^{*}}^{\mathcal{V} \mathcal{W}^{\perp}} & =\left\{\pi_{\mathcal{V}, \mathcal{W}^{\perp}} S_{\mathbf{W}, \mathbf{w}}^{\dagger} T_{\mathbf{W}, \mathbf{w}}+B\left(I_{K_{\mathcal{W}}}-T_{\mathbf{W}, \mathbf{w}}^{*} S_{\mathbf{W}, \mathbf{w}}^{\dagger} T_{\mathbf{W}, \mathbf{w}}\right): B \in L\left(K_{\mathcal{W}}, \mathcal{V}\right)\right\} \\
& =\left\{\pi_{\mathcal{V}, \mathcal{W}^{\perp}} S_{\mathbf{W}, \mathbf{w}}^{\dagger} T_{\mathbf{W}, \mathbf{w}}+B: B \in L\left(K_{\mathcal{W}}, \mathcal{V}\right) \text { and } B T_{\mathbf{W}, \mathbf{w}}^{*}=0\right\}
\end{aligned}
$$


In addition, by [5, Lemma 4],

$$
\begin{aligned}
\mathfrak{L}_{T_{\mathbf{W}, \mathbf{w}}}^{\mathcal{V}, \mathcal{W}^{\perp}} & =\left\{B\left(T_{\mathbf{W}, \mathbf{w}}^{*} B\right)^{\dagger}: B \in L\left(\mathcal{H}, K_{\mathcal{W}}\right) \text { and } \operatorname{Im}(B)=\mathcal{V}\right\} \\
& =\left\{\pi_{\mathcal{V}, \mathcal{W}^{\perp}}\left(B T_{\mathbf{W}, \mathbf{w}}^{*}\right)^{\dagger} B: B \in L\left(K_{\mathcal{W}}, \mathcal{H}\right), \overline{\operatorname{Im}(B)}=\operatorname{Im}(B) \text { and } K_{\mathcal{W}}=\operatorname{Ker}(H) \oplus \operatorname{Im}\left(T_{\mathbf{W}, \mathbf{w}}^{*}\right)\right\} .
\end{aligned}
$$

As a consequence of Theorem 6.3 we obtain the following alternative characterization of component preserving oblique dual fusion frames.

Theorem 6.7. Let $\mathcal{W}$ and $\mathcal{V}$ be finite-dimensional subspaces of the Hilbert space $\mathcal{H}$ such that $\mathcal{H}=\mathcal{V} \oplus \mathcal{W}^{\perp}$. Assume $(\mathbf{W}, \mathbf{w})$ is a fusion frame for $\mathcal{W}$. Then the $Q$-component preserving oblique dual fusion frames of $(\mathbf{W}, \mathbf{w})$ on $\mathcal{V}$ are the families

$$
\left\{\left(V_{i}, v_{i}\right)\right\}_{i \in I}=\left\{\left(\pi_{\mathcal{V}, \mathcal{W}^{\perp}}\left(H T_{\mathbf{W}, \mathbf{w}}^{*}\right)^{\dagger} Z_{i}, v_{i}\right)\right\}_{i \in I},
$$

where $(\mathbf{Z}, \mathbf{w})$ is a fusion frame sequence that satisfies $H T_{\mathbf{W}, \mathbf{w}}^{*}=T_{\mathbf{Z}, \mathbf{w}}\left(H M_{i} T_{\mathbf{W}, \mathbf{w}}^{*}\right)_{i \in I}$, for some $H \in$ $L\left(K_{\mathcal{W}}, \mathcal{H}\right)$ with $\overline{\operatorname{Im}(H)}=\operatorname{Im}(H)$ and $\operatorname{Ker}(H) \oplus \operatorname{Im}\left(T_{\mathbf{W}, \mathbf{w}}^{*}\right)=K_{\mathcal{W}}$ and $Q=Q_{\pi_{\mathcal{V}, \mathcal{W} \perp}\left(H T_{\mathbf{W}, \mathbf{w}}^{*}\right)^{\dagger} H, \mathbf{v}}$. Also,

$$
\left\{\left(V_{i}, v_{i}\right)\right\}_{i \in I}=\left\{\left(B\left(T_{\mathbf{W}, \mathbf{w}}^{*} B\right)^{\dagger} \pi_{\operatorname{Im}\left(T_{\mathbf{W}, \mathbf{w}}^{*}\right), \mathcal{S}} M_{i} K_{\mathcal{W}}, v_{i}\right)\right\}_{i \in I},
$$

where $B \in L\left(K_{\mathcal{W}}, \mathcal{H}\right)$ is such that $\operatorname{Im}(B)=\mathcal{V}$, and $\mathcal{S}$ is a subspace of $K_{\mathcal{W}}$ such that $K_{\mathcal{W}}=$ $\operatorname{Im}\left(T_{\mathbf{W}, \mathbf{w}}^{*}\right) \oplus \mathcal{S}$.

Proof. By Theorem 6.3 and Remark 6.6 the $Q$-oblique component preserving oblique dual fusion frames of $(\mathbf{W}, \mathbf{w})$ on $\mathcal{V}$ are

$$
\left\{\left(V_{i}, v_{i}\right)\right\}_{i \in I}=\left\{\left(\pi_{\mathcal{V}, \mathcal{W}^{\perp}}\left(H T_{\mathbf{W}, \mathbf{w}}^{*}\right)^{\dagger} H M_{i}\left(K_{\mathcal{W}}\right), v_{i}\right)\right\}_{i \in I},
$$

where $H \in L\left(K_{\mathcal{W}}, \mathcal{H}\right)$ is such that $\overline{\operatorname{Im}(H)}=\operatorname{Im}(H)$ and $K_{\mathcal{W}}=\operatorname{Ker}(H) \oplus \operatorname{Im}\left(T_{\mathbf{W}, \mathbf{w}}^{*}\right)$. Taking $Z_{i}=H M_{i}\left(K_{\mathcal{W}}\right)$ we get (6.1).

From Remark 6.6. $\pi_{\mathcal{V}, \mathcal{W}^{\perp}}=R T_{\mathbf{W}, \mathbf{w}}^{*}$, where $R=B\left(T_{\mathbf{W}, \mathbf{w}}^{*} B\right)^{\dagger}$ with $B \in L\left(\mathcal{H}, K_{\mathcal{W}}\right)$ and $\operatorname{Im}(B)=$ $\mathcal{V}$. So we can write, by (6.1),

$$
V_{i}=R T_{\mathbf{W}, \mathbf{w}}^{*}\left(H T_{\mathbf{W}, \mathbf{w}}^{*}\right)^{\dagger} H M_{i}\left(K_{\mathcal{W}}\right)=R \pi_{\operatorname{Im}\left(T_{\mathbf{W}, \mathbf{w}}^{*}\right), \mathcal{S}} M_{i}\left(K_{\mathcal{W}}\right),
$$

where $\mathcal{S}=\operatorname{Ker}(H)$.

We will show now that we can construct component preserving oblique dual fusion frame systems from a given fusion frame for a closed subspace of $\mathcal{H}$ via local dual frames and an oblique left inverse of its analysis operator. 
Proposition 6.8. Let $\mathcal{V}$ and $\mathcal{W}$ be two closed subspaces of $\mathcal{H}$ such that $\mathcal{H}=\mathcal{V} \oplus \mathcal{W}^{\perp}$. Let $(\mathbf{W}, \mathbf{w})$ be a fusion frame for $\mathcal{W}, A \in \mathfrak{L}_{T_{\mathbf{W}, \mathbf{w}}^{*}}^{\mathcal{V} \mathcal{W}^{\perp}}$ and $\mathbf{v}$ be a collection of weights such that $\inf _{i \in I} v_{i}>0$. For each $i \in I$ let $\left\{f_{i, l}\right\}_{l \in L_{i}}$ and $\left\{\tilde{f}_{i, l}\right\}_{l \in L_{i}}$ be dual frames for $W_{i}, \beta_{i}$ upper frame bound of $\left\{f_{i, l}\right\}_{l \in L_{i}}$ for each $i \in I$ such that $\sup _{i \in I} \beta_{i}<\infty, \tilde{\alpha}_{i}$ and $\tilde{\beta}_{i}$ frame bounds of $\left\{\tilde{f}_{i, l}\right\}_{l \in L_{i}}$ for each $i \in I$ such that $\sup _{i \in I} \widetilde{\beta}_{i}<\infty, \mathcal{G}_{i}=\left\{\frac{1}{v_{i}} A\left(\chi_{i}(j) \tilde{f}_{i, l}\right)_{j \in I}\right\}_{l \in L_{i}}$ and $V_{i}=\overline{\operatorname{span}} \mathcal{G}_{i}$. Then

(1) $\mathcal{G}_{i}$ is a frame for $V_{i}$ with frame bounds $\left\|A^{\dagger}\right\|^{-2} \frac{\widetilde{\alpha}_{i}}{v_{i}^{2}}$ and $\|A\|^{2} \frac{\widetilde{\beta}_{i}}{v_{i}^{2}}$.

$(2)(\mathbf{V}, \mathbf{v}, \mathcal{G})$ is a component preserving $Q_{A, \mathbf{v}}$-oblique dual fusion frame system of $(\mathbf{W}, \mathbf{w}, \mathcal{F})$ on $\mathcal{V}$.

Proof. By [4, Proposition 5.3.1], (1) holds.

Let $g \in \mathcal{H}$. We have $\sum_{i \in I} \sum_{l \in L_{i}}\left|\left\langle g, A\left(\chi_{i}(j) \tilde{f}_{i, l}\right)_{j \in I}\right\rangle\right|^{2}=\sum_{i \in I} \sum_{l \in L_{i}}\left|\left\langle A^{*} g,\left(\chi_{i}(j) \tilde{f}_{i, l}\right)_{j \in I}\right\rangle\right|^{2} \leq$ $\sum_{i \in I} \widetilde{\beta}_{i}\left\|M_{i} A^{*} g\right\|^{2} \leq \sup _{i \in I} \widetilde{\beta}_{i} \sum_{i \in I}\left\|M_{i} A^{*} g\right\|^{2}=\left\|A^{*} g\right\|^{2} \sup _{i \in I} \widetilde{\beta}_{i} \leq\|g\|^{2}\|A\|^{2} \sup _{i \in I} \widetilde{\beta}_{i}$. As a consequence of Theorem 2.9. $(\mathbf{V}, \mathbf{v}, \mathcal{G})$ is a Bessel fusion system for $\mathcal{H}$ with upper frame bound $\|A\|^{2} \sup _{i \in I} \widetilde{\beta}_{i}$.

If $\left(h_{i}\right)_{i \in I} \in K_{\mathcal{W}}$, then $Q_{A, \mathbf{v}}$ is a well defined bounded operator with $\left\|Q_{A, \mathbf{v}}\right\| \leq \frac{\|A\|^{2}}{\inf _{i \in I} v_{i}^{2}}$ and $Q_{A, \mathbf{v}}\left(h_{i}\right)_{i \in I}=\left(\frac{1}{v_{i}} A M_{i}\left(h_{j}\right)_{j \in I}\right)_{i \in I}=\left(\frac{1}{v_{i}} A M_{i}\left(\sum_{l \in L_{j}}<h_{j}, f_{j}^{l}>\tilde{f}_{j}^{l}\right)_{j \in I}\right)_{i \in I}=\left(\sum_{l \in L_{i}}<h_{i}, f_{i, l}>\right.$ $\left.\frac{1}{v_{i}} A\left(\chi_{i}(j) \tilde{f}_{i, l}\right)_{j \in I}\right)_{i \in I}=C_{\mathcal{G}} C_{\mathcal{F}}^{*}\left(h_{i}\right)_{i \in I}$. Hence (3) follows from (1), (2) and Lemma 6.2.

The next proposition presents a way to construct component preserving oblique dual fusion frame systems from a given frame for a subspace, using an oblique left inverse of its analysis operator.

Proposition 6.9. Let $\mathcal{V}$ and $\mathcal{W}$ be two closed subspaces of $\mathcal{H}$ such that $\mathcal{H}=\mathcal{V} \oplus \mathcal{W}^{\perp}$. Let $\mathbf{w}$ and $\mathbf{v}$ be two collections of weights such that $\inf _{i \in I} v_{i}>0$. Let $\mathbf{w} \mathcal{F}$ be a frame for $\mathcal{W}$ with local upper frame bound $\beta_{i}$ for each $i \in I$ such that $\sup _{i \in I} \beta_{i}<\infty, A \in \mathfrak{L}_{T_{\mathbf{w} \mathcal{F}}^{*}}^{\mathcal{V} \mathcal{W}^{\perp}}$ and $\left\{\left\{e_{i, l}\right\}_{l \in L_{i}}\right\}_{i \in I}$ be the standard basis for $\oplus_{i \in I} \ell^{2}\left(L_{i}\right)$. For each $i \in I$, set $W_{i}=\overline{\operatorname{span}}\left\{f_{i, l}\right\}_{l \in L_{i}}$ and $V_{i}=\overline{\operatorname{span}}\left\{\frac{1}{v_{i}} A e_{i, l}\right\}_{l \in L_{i}}$. Let $\mathcal{G}=\left\{\left\{\frac{1}{v_{i}} A e_{i, l}\right\}_{l \in L_{i}}\right\}_{i \in I}$. Then

(1) $\left\{\frac{1}{v_{i}} A e_{i, l}\right\}_{l \in L_{i}}$ is a frame for $V_{i}$ with frame bounds $\frac{\left\|A^{\dagger}\right\|^{-2}}{v_{i}^{2}}$ and $\frac{\|A\|^{2}}{v_{i}^{2}}$.

$(2)(\mathbf{V}, \mathbf{v}, \mathcal{G})$ is an oblique dual fusion frame system of $(\mathbf{W}, \mathbf{w}, \mathcal{F})$ on $\mathcal{V}$.

Proof. Part (1) is a consecuence of [4, Proposition 5.3.1]. 
If $g \in \mathcal{H}, \sum_{i \in I} \sum_{l \in L_{i}}\left|\left\langle g, A e_{i, l}\right\rangle\right|^{2}=\sum_{i \in I} \sum_{l \in L_{i}}\left|\left\langle A^{*} g, e_{i, l}\right\rangle\right|^{2}=\left\|A^{*} g\right\|^{2} \leq\|A\|^{2}\|g\|^{2}$. By Theorem 2.9, $(\mathbf{V}, \mathbf{v}, \mathcal{G})$ is a Bessel fusion system for $\mathcal{H}$ with upper frame bound $\|A\|^{2}$.

By [6. Lemma B.1], $\mathbf{v} \mathcal{G}$ is an oblique dual frame of $\mathbf{w} \mathcal{F}$ on $\mathcal{V}$. So, part (2) follows from Theorem 4.4 ,

\section{The Canonical oblique dual fusion frame}

Let $(\mathbf{W}, \mathbf{w})$ be a fusion frame for $\mathcal{W}$. Let $A=\pi_{\mathcal{V}, \mathcal{W}^{\perp}} S_{\mathbf{W}, \mathbf{w}}^{\dagger} T_{\mathbf{W}, \mathbf{w}} \in \mathfrak{L}_{T_{\mathbf{W}, \mathbf{w}}^{*}}^{\mathcal{V}, \mathcal{W}^{\perp}}$ and $\mathbf{v}$ be a sequence of weights such that $\left(\pi_{\mathcal{V}, \mathcal{W}^{\perp}} S_{\mathbf{W}, \mathbf{w}}^{\dagger} \mathbf{W}, \mathbf{v}\right)$ is a Bessel fusion sequence for $\mathcal{V}$. Assume that $Q_{A, \mathbf{v}}: K_{\mathcal{W}} \rightarrow \oplus_{i \in I} \pi_{\mathcal{V}, \mathcal{W}^{\perp}} S_{\mathbf{W}, \mathbf{w}}^{\dagger} W_{i}$ given by $Q_{A, \mathbf{v}}\left(f_{i}\right)_{i \in I}=\left(\frac{w_{i}}{v_{i}} \pi_{\mathcal{V}, \mathcal{W}^{\perp}} S_{\mathbf{W}, \mathbf{w}}^{\dagger} f_{i}\right)_{i \in I}$ is a well defined bounded operator (see [11, Remark 3.6]). By Lemma 6.2 $\left(\pi_{\mathcal{V}, \mathcal{W}^{\perp}} S_{\mathbf{W}, \mathbf{w}}^{\dagger} \mathbf{W}, \mathbf{v}\right)$ is a $Q_{A, \mathbf{v}}$-component preserving dual of $(\mathbf{W}, \mathbf{w})$ on $\mathcal{V}$. In particular we can take $\mathbf{v}=\mathbf{w}$. In fact, $\left(S_{\mathbf{W}, \mathbf{w}}^{\dagger} \mathbf{W}, \mathbf{w}\right)$ is a $Q_{S_{\mathbf{W}, \mathbf{w}}^{\dagger} T_{\mathbf{W}, \mathbf{w}}, \mathbf{w}}$-component preserving dual of $(\mathbf{W}, \mathbf{w})$ (see [11, Example 3.7]). Then, by Corollary 5.4. $\left(\pi_{\mathcal{V}, \mathcal{W} \perp} S_{\mathbf{W}, \mathbf{w}}^{\dagger} \mathbf{W}, \mathbf{w}\right)$ is a $Q_{A, \mathbf{w}}$-oblique component preserving dual of $(\mathbf{W}, \mathbf{w})$ on $\mathcal{V}$.

Given $\mathbf{v}$ we will refer to this dual as the canonical oblique dual with weights $\mathbf{v}$ and to

$$
Q_{\pi_{\mathcal{V}, \mathcal{W} \perp} S_{\mathrm{w}, \mathrm{w}} T_{\mathbf{W}, \mathbf{w}, \mathbf{v}}}^{*} T_{\pi_{\mathcal{V}, \mathcal{W}^{\perp}} S_{\mathbf{W}, \mathbf{w}} \mathbf{W}, \mathbf{v}}^{\dagger} f=T_{\mathbf{W}, \mathbf{w}}^{*} S_{\mathbf{W}, \mathbf{w}}^{\dagger} \pi_{\mathcal{W}, \mathcal{V} \perp} f
$$

as the oblique fusion frame coefficients of $f \in \mathcal{H}$ with respect to $(\mathbf{W}, \mathbf{w})$ on $\mathcal{V}$. We note that if $\mathcal{V}=\mathcal{W}$, the canonical oblique dual fusion frames reduce to the canonical dual fusion frames as defined in [11, 12].

Furthermore, if in Definition $3.6(\mathbf{V}, \mathbf{v})$ is a canonical oblique dual fusion frame of $(\mathbf{W}, \mathbf{w})$ we say that $(\mathbf{V}, \mathbf{v}, \mathcal{G})$ is a canonical oblique dual fusion frame system of $(\mathbf{W}, \mathbf{w}, \mathcal{F})$.

The theorem below follows from Theorem 6.3 It gives a characterizations of canonical oblique dual fusion frames and can be proved in a similar way as [5, Theorem 2].

Theorem 7.1. Let $\mathcal{V}$ and $\mathcal{W}$ be two finite-dimensional subspaces of $\mathcal{H}$ such that $\mathcal{H}=\mathcal{V} \oplus \mathcal{W}^{\perp}$. Assume $(\mathbf{W}, \mathbf{w})$ is a fusion frame for a subspace $\mathcal{W} \subseteq \mathcal{H}$. Then the canonical $Q$-oblique dual fusion frames of $(\mathbf{W}, \mathbf{w})$ on $\mathcal{V}$ are

$$
\left\{\left(V_{i}, v_{i}\right)\right\}_{i \in I}=\left\{\left(\pi_{\mathcal{V}, \mathcal{W}^{\perp}}\left(H T_{\mathbf{W}, \mathbf{w}}^{*}\right)^{\dagger} Z_{i}, v_{i}\right)\right\}_{i \in I},
$$

where $(\mathbf{Z}, \mathbf{w})$ is a fusion frame sequence that satisfies $H T_{\mathbf{W}, \mathbf{w}}^{*}=T_{\mathbf{Z}, \mathbf{w}}\left(H M_{i} T_{\mathbf{W}, \mathbf{w}}^{*}\right)_{i \in I}$, for some $H \in L\left(K_{\mathcal{W}}, \mathcal{H}\right)$ with $\operatorname{Ker}(H)=\operatorname{Ker}\left(T_{\mathbf{W}, \mathbf{w}}\right)$ and $Q=Q_{\pi_{\mathcal{V}, \mathcal{W} \perp}\left(H T_{\mathbf{W}, \mathbf{w}}^{*}\right)^{\dagger} H, \mathbf{v}}$. Also

$$
\left\{\left(V_{i}, v_{i}\right)\right\}_{i \in I}=\left\{\left(B\left(T_{\mathbf{W}, \mathbf{w}}^{*} B\right)^{\dagger} M_{i}\left(K_{\mathcal{W}}\right), v_{i}\right)\right\}_{i \in I},
$$


where $B \in L\left(K_{\mathcal{W}}, \mathcal{H}\right)$ is such that $\operatorname{Im}(B)=\mathcal{V}$.

The following lemma implies that oblique fusion frame coefficients are those which have minimal norm among all other coefficients.

Lemma 7.2. Let $\mathcal{W}$ and $\mathcal{V}$ be two closed subspaces of $\mathcal{H}$ such that $\mathcal{H}=\mathcal{V} \oplus \mathcal{W}^{\perp}$. Let $(\mathbf{W}, \mathbf{w})$ be a fusion frame for $\mathcal{W}$ and $f \in \mathcal{H}$. For all $\left(f_{i}\right)_{i \in I} \in K_{\mathcal{W}}$ satisfying $T_{\mathbf{W}, \mathbf{w}}\left(f_{i}\right)_{i \in I}=\pi_{\mathcal{W}, \mathcal{V} \perp} f$, we have

$$
\left\|\left(f_{i}\right)_{i \in I}\right\|^{2}=\left\|T_{\mathbf{W}, \mathbf{w}}^{*} S_{\mathbf{W}, \mathbf{w}}^{\dagger} \pi_{\mathcal{W}, \mathcal{V} \perp} f\right\|^{2}+\left\|\left(f_{i}\right)_{i \in I}-T_{\mathbf{W}, \mathbf{w}}^{*} S_{\mathbf{W}, \mathbf{w}}^{\dagger} \pi_{\mathcal{W}, \mathcal{V} \perp} f\right\|^{2} .
$$

Proof. Suppose that $\left(f_{i}\right)_{i \in I} \in K_{\mathcal{W}}$ satisfies $T_{\mathbf{W}, \mathbf{w}}\left(f_{i}\right)_{i \in I}=\pi_{\mathcal{W}, \mathcal{V} \perp} f$. Then

$$
\left(f_{i}\right)_{i \in I}-T_{\mathbf{W}, \mathbf{w}}^{*} S_{\mathbf{W}, \mathbf{w}}^{\dagger} \pi_{\mathcal{W}, \mathcal{V}} f \in \operatorname{Ker}\left(T_{\mathbf{W}, \mathbf{w}}\right)=\operatorname{Im}\left(T_{\mathbf{W}, \mathbf{w}}^{*}\right)^{\perp} .
$$

Since $T_{\mathbf{W}, \mathbf{w}}^{*} S_{\mathbf{W}, \mathbf{w}}^{\dagger} \pi_{\mathcal{W}, \mathcal{V} \perp} f \in \operatorname{Im}\left(T_{\mathbf{W}, \mathbf{w}}^{*}\right)$, the conclusion follows.

Remark 7.3. Let $\mathcal{W}$ and $\mathcal{V}$ be two closed subspaces of $\mathcal{H}$ such that $\mathcal{H}=\mathcal{V} \oplus \mathcal{W}^{\perp}$. Let $(\mathbf{W}, \mathbf{w})$ be a fusion frame for $\mathcal{W}$ and $(\mathbf{V}, \mathbf{v})$ be a fusion frame for $\mathcal{V}$.

Since $\mathcal{H}=\mathcal{V} \oplus \mathcal{W}^{\perp}$, the operator $T_{\mathbf{W}, \mathbf{w}}^{*} T_{\mathbf{V}, \mathbf{v}}: K_{\mathcal{V}} \rightarrow K_{\mathcal{W}}$ given by $T_{\mathbf{W}, \mathbf{w}}^{*} T_{\mathbf{V}, \mathbf{v}}\left(g_{i}\right)_{i \in I}=$ $\sum_{i \in I} v_{i} T_{\mathbf{W}, \mathbf{w}}^{*} g_{i}=\left(w_{l} \sum_{i \in I} v_{i} \pi_{W_{l}} g_{i}\right)_{l \in I}$, satisfies $\operatorname{Ker}\left(T_{\mathbf{W}, \mathbf{w}}^{*} T_{\mathbf{V}, \mathbf{v}}\right)=\operatorname{Ker}\left(T_{\mathbf{V}, \mathbf{v}}\right)$ and $\operatorname{Im}\left(T_{\mathbf{W}, \mathbf{w}}^{*} T_{\mathbf{V}, \mathbf{v}}\right)=$ $\operatorname{Im}\left(T_{\mathbf{W}, \mathbf{w}}^{*}\right)$.

If $f \in \mathcal{W}^{\perp}=\operatorname{Ker}\left(T_{\mathbf{W}, \mathbf{w}}^{*}\right), T_{\mathbf{V}, \mathbf{v}}\left(T_{\mathbf{W}, \mathbf{w}}^{*} T_{\mathbf{V}, \mathbf{v}}\right)^{\dagger} T_{\mathbf{W}, \mathbf{w}}^{*} f=0$. If $g \in \mathcal{V}=\operatorname{Im}\left(T_{\mathbf{V}, \mathbf{v}}\right)$, there exists $\left(g_{i}\right)_{i \in I} \in K_{\mathcal{V}}$ such that $g=T_{\mathbf{V}, \mathbf{v}}\left(g_{i}\right)_{i \in I}$. Then $T_{\mathbf{V}, \mathbf{v}}\left(T_{\mathbf{W}, \mathbf{w}}^{*} T_{\mathbf{V}, \mathbf{v}}\right)^{\dagger} T_{\mathbf{W}, \mathbf{w}}^{*} g=T_{\mathbf{V}, \mathbf{v}} \pi_{\operatorname{Ker}\left(T_{\mathbf{V}, \mathbf{v}}\right)^{\perp}}\left(g_{i}\right)_{i \in I}=$ $T_{\mathbf{V}, \mathbf{v}}\left(g_{i}\right)_{i \in I}=g$. Thus $T_{\mathbf{V}, \mathbf{v}}\left(T_{\mathbf{W}, \mathbf{w}}^{*} T_{\mathbf{V}, \mathbf{v}}\right)^{\dagger} T_{\mathbf{W}, \mathbf{w}}^{*}=\pi_{\mathcal{V}, \mathcal{W}^{\perp}}$ and consequently, $(\mathbf{V}, \mathbf{v})$ is a $\left(T_{\mathbf{W}, \mathbf{w}}^{*} T_{\mathbf{V}, \mathbf{v}}\right)^{\dagger}-$ oblique dual fusion frame of $(\mathbf{W}, \mathbf{w})$ on $\mathcal{V}$.

This shows that given $(\mathbf{W}, \mathbf{w})$ a fusion frame for $\mathcal{W}$ and $(\mathbf{V}, \mathbf{v})$ a fusion frame for $\mathcal{V}$ we can always do the analysis with one of them and the synthesis with the other leading to a consistent reconstruction. Note that this happens in the general framework of Definition 3.2 where we do not impose any additional condition on $Q$.

We note that the component preserving oblique duals associate with $T_{\mathbf{V}, \mathbf{v}}\left(T_{\mathbf{W}, \mathbf{w}}^{*} T_{\mathbf{V}, \mathbf{v}}\right)^{\dagger} \in$ $\mathfrak{L}_{T_{\mathbf{W}, \mathbf{w}}^{*}}^{\mathcal{V}, \mathcal{W}^{\perp}}$ obtained applying Lemma 6.2 are the canonical ones. To see this we will prove that $T_{\mathbf{V}, \mathbf{v}}\left(T_{\mathbf{W}, \mathbf{w}}^{*} T_{\mathbf{V}, \mathbf{v}}\right)^{\dagger}=\pi_{\mathcal{V}, \mathcal{W}} S_{\mathbf{W}, \mathbf{w}}^{\dagger} T_{\mathbf{W}, \mathbf{w}}$. We have on one hand

$$
T_{\mathbf{V}, \mathbf{v}}\left(T_{\mathbf{W}, \mathbf{w}}^{*} T_{\mathbf{V}, \mathbf{v}}\right)^{\dagger} T_{\mathbf{W}, \mathbf{w}}^{*}=\pi_{\mathcal{V}, \mathcal{W}^{\perp}} S_{\mathbf{W}, \mathbf{w}}^{\dagger} T_{\mathbf{W}, \mathbf{w}} T_{\mathbf{W}, \mathbf{w}}^{*}=\pi_{\mathcal{V}, \mathcal{W}^{\perp}}
$$

Let now $\left(g_{i}\right)_{i \in I} \in \operatorname{Im}\left(T_{\mathbf{W}, \mathbf{w}}^{*}\right)^{\perp}=\operatorname{Ker}\left(T_{\mathbf{W}, \mathbf{w}}\right)=\operatorname{Ker}\left(\left(T_{\mathbf{W}, \mathbf{w}}^{*} T_{\mathbf{V}, \mathbf{v}}\right)^{\dagger}\right)$. Then $T_{\mathbf{V}, \mathbf{v}}\left(T_{\mathbf{W}, \mathbf{w}}^{*} T_{\mathbf{V}, \mathbf{v}}\right)^{\dagger}\left(g_{i}\right)_{i \in I}=$ $\pi_{\mathcal{V}, \mathcal{W} \perp} S_{\mathbf{W}, \mathbf{w}}^{\dagger} T_{\mathbf{W}, \mathbf{w}}\left(g_{i}\right)_{i \in I}=0$ 
7.1. Existence of non-canonical oblique dual fusion frames. A Bessel fusion sequence $(\mathbf{W}, \mathbf{w})$ is a Riesz fusion basis for $\mathcal{W}$ if and only if $T_{\mathbf{W}, \mathbf{w}}$ is injective, or equivalently, $S_{\mathbf{W}, \mathbf{w}}^{\dagger} T_{\mathbf{W}, \mathbf{w}}$ is the unique element in $\mathfrak{L}_{T_{\mathbf{W}, \mathbf{w}}^{*}}^{\mathcal{W}}$. In this case, by Proposition $2.3 \pi \pi_{\mathcal{V}, \mathcal{W} \perp} S_{\mathbf{W}, \mathbf{w}}^{\dagger} T_{\mathbf{W}, \mathbf{w}}$ is the unique element in $\mathfrak{L}_{T_{\mathbf{W}, \mathbf{w}}^{*}}^{\mathcal{V}, \mathcal{W}^{\perp}}$. So, by Lemma 6.1] if $(\mathbf{W}, \mathbf{w})$ is a Riesz fusion basis for $\mathcal{W}$ the only component preserving duals of $(\mathbf{W}, \mathbf{w})$ are $\left(\pi_{\mathcal{V}, \mathcal{W} \perp} S_{\mathbf{W}, \mathbf{w}}^{\dagger} \mathbf{W}, \mathbf{v}\right)$. It is easy to see that if $Q_{\pi_{\mathcal{V}, \mathcal{W} \perp} S_{\mathbf{W}, \mathbf{w}}^{\dagger} T_{\mathbf{W}, \mathbf{w}, \mathbf{v}}}$ is well defined and bounded for the weights $\mathbf{v}$, this component preserving oblique dual coincides with the canonical one with weights $\mathbf{v}$, i.e. the operator $Q$ for this dual is $Q_{\pi_{\mathcal{V}, \mathcal{W} \perp} S_{\mathrm{W}, \mathbf{w}}^{\dagger} T_{\mathbf{W}, \mathbf{w}}, \mathbf{v}}$. We also have:

Proposition 7.4. Let $\mathcal{W}$ and $\mathcal{V}$ be two closed subspaces of $\mathcal{H}$ such that $\mathcal{H}=\mathcal{V} \oplus \mathcal{W}^{\perp}$. Let $(\mathbf{W}, \mathbf{w})$ be a Riesz fusion basis for $\mathcal{W}$ and $\mathbf{v}$ a family of weights. The following assertions hold:

(1) $\left(\pi_{\mathcal{V}, \mathcal{W} \perp} S_{\mathbf{W}, \mathbf{w}}^{\dagger} \mathbf{W}, \mathbf{w}\right)$ is a Riesz fusion basis for $\mathcal{V}$

(2) If $(\mathbf{V}, \mathbf{v})$ is a Riesz fusion basis for $\mathcal{V}$, then $T_{\mathbf{V}, \mathbf{v}}^{*} T_{\mathbf{W}, \mathbf{w}}$ is invertible.

(3) Let $(\mathbf{V}, \mathbf{v})$ be a block-diagonal oblique dual fusion frame of $(\mathbf{W}, \mathbf{w})$ on $\mathcal{V}$. Then, for each $i \in I, \pi_{\mathcal{V}, \mathcal{W} \perp} S_{\mathbf{W}, \mathbf{w}}^{\dagger} W_{i} \subseteq V_{i}$.

(4) If $(\mathbf{V}, \mathbf{v})$ is a Riesz fusion basis for $\mathcal{V}$ which is a block-diagonal oblique dual fusion frame of $(\mathbf{W}, \mathbf{w})$ on $\mathcal{V}$, then $V_{i}=\pi_{\mathcal{V}, \mathcal{W} \perp} S_{\mathbf{W}, \mathbf{w}}^{\dagger} W_{i}$ for $i \in I$

Proof. (1) Let $\left(f_{i}\right)_{i \in I} \in K_{\mathcal{W}}$. Since $(\mathbf{W}, \mathbf{w})$ is a Riesz fusion basis for $\mathcal{W}$, there exists $f \in \mathcal{H}$ such that $\left(f_{i}\right)_{i \in I}=T_{\mathbf{W}, \mathbf{w}}^{*} f$. Then

$$
\begin{aligned}
T_{\pi_{\mathcal{V}, \mathcal{W} \perp} S_{\mathbf{W}, \mathbf{w}}^{\dagger} \mathbf{W}, \mathbf{w}}\left(\pi_{\mathcal{V}, \mathcal{W}^{\perp}} S_{\mathbf{W}, \mathbf{w}}^{\dagger} f_{i}\right)_{i \in I} & =\pi_{\mathcal{V}, \mathcal{W}^{\perp}} S_{\mathbf{W}, \mathbf{w}}^{\dagger} T_{\mathbf{W}, \mathbf{w}}\left(f_{i}\right)_{i \in I} \\
& =\pi_{\mathcal{V}, \mathcal{W}^{\perp}} S_{\mathbf{W}, \mathbf{w}}^{\dagger} T_{\mathbf{W}, \mathbf{w}} T_{\mathbf{W}, \mathbf{w}}^{*} f=\pi_{\mathcal{V}, \mathcal{W}^{\perp}} \pi_{\mathcal{W}} f=\pi_{\mathcal{V}, \mathcal{W} \perp} f
\end{aligned}
$$

Thus $T_{\pi_{\mathcal{V}, \mathcal{W}} \perp S_{\mathbf{W}, \mathbf{w}}^{\dagger} \mathbf{W}, \mathbf{w}}\left(\pi_{\mathcal{V}, \mathcal{W} \perp} S_{\mathbf{W}, \mathbf{w}}^{\dagger} f_{i}\right)_{i \in I}=0$ if and only if $f \in \mathcal{W}^{\perp}=\operatorname{Ker}\left(T_{\mathbf{W}, \mathbf{w}}^{*}\right)$, that is, $\left(f_{i}\right)_{i \in I}=0$. It follows that $T_{\pi_{\mathcal{V}, \mathcal{W} \perp} S_{\mathbf{W}, \mathbf{w}}^{\dagger} \mathbf{W}, \mathbf{w}}$ is injective, or equivalently, $\left(\pi_{\mathcal{V}, \mathcal{W} \perp} S_{\mathbf{W}, \mathbf{w}}^{\dagger} \mathbf{W}, \mathbf{w}\right)$ is a Riesz fusion basis for $\mathcal{V}$.

(2) Let $\left(f_{i}\right)_{i \in I} \in K_{\mathcal{W}}$ such that $T_{\mathbf{V}, \mathbf{v}}^{*} T_{\mathbf{W}, \mathbf{w}}\left(f_{i}\right)_{i \in I}=0$. So, $T_{\mathbf{W}, \mathbf{w}}\left(f_{i}\right)_{i \in I} \in \operatorname{Ker}\left(T_{\mathbf{V}, \mathbf{v}}^{*}\right) \cap$ $\operatorname{Im}\left(T_{\mathbf{W}, \mathbf{w}}\right)=\mathcal{V}^{\perp} \cap \mathcal{W}=\{0\}$. Since $(\mathbf{W}, \mathbf{w})$ is a Riesz fusion basis for $\mathcal{W}$, this implies that $\left(f_{i}\right)_{i \in I}=0$. Therefore, $T_{\mathbf{V}, \mathbf{v}}^{*} T_{\mathbf{W}, \mathbf{w}}$ is injective. In the same manner it results that $\left(T_{\mathbf{V}, \mathbf{v}}^{*} T_{\mathbf{W}, \mathbf{w}}\right)^{*}=$ $T_{\mathbf{W}, \mathbf{w}}^{*} T_{\mathbf{V}, \mathbf{v}}$ is injective. Consequently, $T_{\mathbf{V}, \mathbf{v}}^{*} T_{\mathbf{W}, \mathbf{w}}$ is bijective. 
(3) Let $\left(f_{i}\right)_{i \in I} \in K_{\mathcal{W}}$ and $i \in I$ fixed. From $T_{\mathbf{V}, \mathbf{v}} Q T_{\mathbf{W}, \mathbf{w}}^{*}=\pi_{\mathcal{V}, \mathcal{W} \perp}$ and $T_{\mathbf{W}, \mathbf{w}}^{*} S_{\mathbf{W}, \mathbf{w}}^{\dagger} T_{\mathbf{W}, \mathbf{w}}=I_{K_{\mathcal{W}}}$ (the last equality holds since $\left(T_{\mathbf{W}, \mathbf{w}}^{*}\right)_{\mid \mathcal{W}}: \mathcal{W} \rightarrow K_{\mathcal{W}}$ is bijective and $S_{\mathbf{W}, \mathbf{w}}^{\dagger} T_{\mathbf{W}, \mathbf{w}} T_{\mathbf{W}, \mathbf{w}}^{*}=\pi_{\mathcal{W}}$ ), we obtain

$$
\begin{aligned}
\pi_{\mathcal{V}, \mathcal{W}^{\perp}} S_{\mathbf{W}, \mathbf{w}}^{\dagger} f_{i} & =T_{\mathbf{V}, \mathbf{v}} Q T_{\mathbf{W}, \mathbf{w}}^{*} S_{\mathbf{W}, \mathbf{w}}^{\dagger} T_{\mathbf{W}, \mathbf{w}} M_{i}\left(\chi_{i}(j) \frac{1}{w_{j}} f_{j}\right)_{j \in I} \\
& =T_{\mathbf{V}, \mathbf{v}} Q M_{i}\left(\chi_{i}(j) \frac{1}{w_{j}} f_{j}\right)_{j \in I}=T_{\mathbf{V}, \mathbf{v}} M_{i} Q\left(\chi_{i}(j) \frac{1}{w_{j}} f_{j}\right)_{j \in I} \in V_{i} .
\end{aligned}
$$

So $\pi_{\mathcal{V}, \mathcal{W}^{\perp}} S_{\mathrm{W}, \mathrm{w}}^{\dagger} W_{i} \subseteq V_{i}$.

(44) By (33), $\pi_{\mathcal{V}, \mathcal{W}^{\perp}} S_{\mathrm{W}, \mathbf{w}}^{\dagger} W_{i} \subseteq V_{i}$ for each $i \in I$. Suppose that there exists $i_{0} \in I$ such that $\pi_{\mathcal{V}, \mathcal{W}^{\perp}} S_{\mathbf{W}, \mathbf{w}}^{\dagger} W_{i_{0}} \subset V_{i_{0}}$. Set $\{0\} \neq U_{i_{0}} \subset V_{i_{0}}$ such that $V_{i_{0}}=\pi_{\mathcal{V}, \mathcal{W} \perp} S_{\mathbf{W}, \mathbf{w}}^{\dagger} W_{i_{0}} \oplus U_{i_{0}}$ Take $0 \neq u_{i_{0}} \in$ $U_{i_{0}}$. By (1), $u_{i_{0}}=\sum_{i \in I} g_{i}$ where $g_{i} \in \pi_{\mathcal{V}, \mathcal{W} \perp} S_{\mathbf{W}, \mathbf{w}}^{\dagger} W_{i}$ for each $i \in I$. Since $\pi_{\mathcal{V}, \mathcal{W}^{\perp}} S_{\mathbf{W}, \mathbf{w}}^{\dagger} W_{i} \subseteq V_{i}$ for each $i \in I$ and $(\mathbf{V}, \mathbf{v})$ is a Riesz fusion basis for $\mathcal{V}, u_{i_{0}}=g_{i_{0}} \in \pi_{\mathcal{V}, \mathcal{W}^{\perp}} S_{\mathbf{W}, \mathbf{w}}^{\dagger} W_{i_{0}} \cap U_{i_{0}}=\{0\}$. This is absurd. Thus the conclusion follows.

Remark 7.5. Let $\mathcal{W}$ and $\mathcal{V}$ be two closed subspaces of $\mathcal{H}$. Let $(\mathbf{W}, \mathbf{w})$ be a fusion Riesz basis for $\mathcal{W}$ and $(\mathbf{V}, \mathbf{v})$ a fusion Riesz basis for $\mathcal{V}$. If $T_{\mathbf{V}, \mathbf{v}}^{*} T_{\mathbf{W}, \mathbf{w}}$ is injective, then $\mathcal{W} \cap \mathcal{V}^{\perp}=\{0\}$. To see this, consider $f \in \mathcal{W} \cap \mathcal{V}^{\perp}$. Since $f \in \mathcal{W}=\operatorname{Im}\left(T_{\mathbf{W}, \mathbf{w}}\right)$, there exists $\left(f_{i}\right)_{i \in I} \in K_{\mathcal{W}}$ such that $f=T_{\mathbf{W}, \mathbf{w}}\left(f_{i}\right)_{i \in I}$. Since $f \in \mathcal{V}^{\perp}=\operatorname{Ker}\left(T_{\mathbf{V}, \mathbf{v}}^{*}\right), T_{\mathbf{V}, \mathbf{v}}^{*} f=0$. Therefore, $T_{\mathbf{V}, \mathbf{v}}^{*} T_{\mathbf{W}, \mathbf{w}}\left(f_{i}\right)_{i \in I}=0$. Taking into account that $T_{\mathbf{V}, \mathbf{v}}^{*} T_{\mathbf{W}, \mathbf{w}}$ is injective, we deduce that $\left(f_{i}\right)_{i \in I}=0$, and then $f=0$.

To prove Proposition 7.8 about the existence of non canonical oblique duals, we will need the following Corollary which is a consequence of the next lemma that generalizes Lemma 5.5.5 in [4, and can be proved in a similar way.

Lemma 7.6. Let $\mathcal{W}$ be a closed subspace of $\mathcal{H} . A$ pair $(\mathbf{W}, \mathbf{w})$ is a fusion frame for $\mathcal{W}$ with bounds $A, B$ if and only if the following conditions are satisfied:

(1) $(\mathbf{W}, \mathbf{w})$ is complete in $\mathcal{W}$

(2) The operator $T_{\mathbf{W}, \mathbf{w}}$ is well defined on $\mathcal{K}_{\mathcal{W}}$ and

$$
A\left\|\left(f_{i}\right)_{i \in I}\right\|^{2} \leq\left\|T_{\mathbf{W}, \mathbf{w}}\left(f_{i}\right)_{i \in I}\right\|^{2} \leq B\left\|\left(f_{i}\right)_{i \in I}\right\|^{2} \forall\left(f_{i}\right)_{i \in I} \in \operatorname{Ker}\left(T_{\mathbf{W}, \mathbf{w}}\right)^{\perp}
$$

Corollary 7.7. Let $\mathcal{W}$ be a closed subspace of $\mathcal{H}$ and $(\mathbf{W}, \mathbf{w})$ be a fusion frame for $\mathcal{W}$. Let $(\widetilde{\mathbf{W}}, \mathbf{w})$ be a sequence such that $\widetilde{W}_{i} \subseteq W_{i}$ for all $i \in I$. Then $(\widetilde{\mathbf{W}}, \mathbf{w})$ is either a fusion frame for $\mathcal{W}$ or incomplete in $\mathcal{W}$. 
Proof. Assume that $(\widetilde{\mathbf{W}}, \mathbf{w})$ is complete in $\mathcal{W}$. Since $\pi_{\widetilde{W}_{i}}=\pi_{\widetilde{W}_{i}} \pi_{W_{i}}$ It is clear that $(\widetilde{\mathbf{W}}, \mathbf{w})$ is a Bessel sequence, so the operator $T_{\widetilde{\mathbf{W}}, \mathbf{w}}$ is well defined on $\mathcal{K}_{\widetilde{\mathcal{W}}}$.

Considering everything inside $\mathcal{K}_{\widetilde{\mathcal{W}}}$ we can decompose

$$
\operatorname{Ker}\left(T_{\widetilde{\mathbf{W}}, \mathbf{w}}\right)^{\perp}=\operatorname{Ker}\left(T_{\mathbf{W}, \mathbf{w}}\right)^{\perp} \oplus\left(\operatorname{Ker}\left(T_{\mathbf{W}, \mathbf{w}}\right)^{\perp}\right)^{\perp}=\operatorname{Ker}\left(T_{\mathbf{W}, \mathbf{w}}\right)^{\perp} \oplus \operatorname{Ker}\left(T_{\mathbf{W}, \mathbf{w}}\right) .
$$

Hence, by Lemma 7.6

$$
\left\|T_{\widetilde{\mathbf{W}}, \mathbf{w}}\left(f_{i}\right)_{i \in I}\right\|^{2} \geq A\left\|\pi_{\operatorname{Ker}\left(T_{\mathbf{W}, \mathbf{w}}\right)^{\perp}}\left(f_{i}\right)_{i \in I}\right\|^{2} \forall\left(f_{i}\right)_{i \in I} \in \operatorname{Ker}\left(T_{\widetilde{\mathbf{W}}, \mathbf{w}}\right)^{\perp} .
$$

Since $\operatorname{span} \bigcup_{i \in I} \widetilde{W}_{i} \subseteq \operatorname{Im}\left(T_{\widetilde{\mathbf{W}}, \mathbf{w}}\right) \subseteq \overline{\operatorname{span}} \bigcup_{i \in I} \widetilde{W}_{i}=\mathcal{W}$, we only have to prove that $\operatorname{Im}\left(T_{\widetilde{\mathbf{W}}, \mathbf{w}}\right)$ is closed. Let $g \in \overline{\operatorname{Im}\left(T_{\widetilde{\mathbf{W}}, \mathbf{w}}\right)}$. Then there exists a sequence $\left(f_{i}\right)_{i \in I}^{n} \in \operatorname{Ker}\left(T_{\mathbf{W}, \mathbf{w}}\right)^{\perp}$ such that $T_{\widetilde{\mathbf{W}}, \mathbf{w}}\left(f_{i}\right)_{i \in I}^{n}$ converges to $g$. By (17.1) $\left(f_{i}\right)_{i \in I}^{n}$ is a Cauchy sequence, so it converges to some $\left(f_{i}\right)_{i \in I}$ in $\mathcal{K}_{\widetilde{\mathcal{W}}}$ which satisfies, by continuity, that $T_{\widetilde{\mathbf{W}}, \mathbf{w}}\left(f_{i}\right)_{i \in I}=g$.

The next proposition shows that if $(\mathbf{W}, \mathbf{w})$ is an overcomplete fusion frame with non trivial subspaces there always exist component preserving oblique dual fusion frames which are not the canonical ones. For $\mathcal{V}=\mathcal{W}$ this result is a generalization of [12, Proposition 3.9] to the infinite dimensional case.

Proposition 7.8. Let $(\mathbf{W}, \mathbf{w})$ be a fusion frame for a closed subspace $\mathcal{W} \subseteq \mathcal{H}$ and let $\mathcal{V}$ be a closed subspace such that $\mathcal{H}=\mathcal{V} \oplus \mathcal{W}^{\perp}$. Let $(\mathbf{W}, \mathbf{w})$ be an overcomplete fusion frame for $\mathcal{W}$ such that $W_{i} \neq\{0\}$ for every $i \in I$. Then there exist component preserving oblique dual fusion frames $(\mathbf{V}, \mathbf{w})$ of $(\mathbf{W}, \mathbf{w})$ different from $\left(\pi_{\mathcal{V}, \mathcal{W}^{\perp}} S_{\mathbf{W}, \mathbf{w}}^{\dagger} \mathbf{W}, \mathbf{w}\right)$.

Proof. Since $(\mathbf{W}, \mathbf{w})$ is not a Riesz fusion basis, there exists $i_{0} \in I$ such that $W_{i_{0}} \cap \overline{\operatorname{span}}\left\{W_{i}: i \neq\right.$ $\left.i_{0}\right\} \neq\{0\}$. Let us first prove that $(\widetilde{\mathbf{W}}, \mathbf{w})$ given by $\widetilde{W}_{i}=W_{i}$ for $i \neq i_{0}$ and $\widetilde{W}_{i_{0}}=W_{i_{0}} \cap\left(W_{i_{0}} \cap\right.$ $\left.\overline{\operatorname{span}}\left\{W_{i}: i \neq i_{0}\right\}\right)^{\perp}$ is a fusion frame for $\mathcal{W}$.

Let us first see that $(\widetilde{\mathbf{W}}, \mathbf{w})$ given by $\widetilde{W}_{i}=W_{i}$ for $i \neq i_{0}$ and $\widetilde{W}_{i_{0}}=W_{i_{0}} \cap\left(\overline{\operatorname{span}}\left\{W_{i}: i \neq\right.\right.$ $\left.\left.i_{0}\right\} \cap W_{i_{0}}\right)^{\perp}$ is a fusion frame for $\mathcal{W}$.

Let $f \in \mathcal{W}$. Then $f=\sum_{i \in I} f_{i}$, with $f_{i} \in W_{i}$ for all $i \in I$. So, $f=\sum_{i \neq i_{0}} f_{i}+f_{i_{0}}=$ $\sum_{i \neq i_{0}} f_{i}+\pi_{\overline{\mathrm{span}}\left\{W_{i}: i \neq i_{0}\right\} \cap W_{i_{0}}}\left(f_{i_{0}}\right)+\pi_{\left(\overline{\operatorname{span}}\left\{W_{i}: i \neq i_{0}\right\} \cap W_{i_{0}}\right)^{\perp}}\left(f_{i_{0}}\right)$.

But then $\pi_{\left(\overline{\operatorname{span}}\left\{W_{i}: i \neq i_{0}\right\} \cap W_{i_{0}}\right)^{\perp}}\left(f_{i_{0}}\right) \in W_{i_{0}}$, hence $\pi_{\left(\overline{\operatorname{span}}\left\{W_{i}: i \neq i_{0}\right\} \cap W_{i_{0}}\right)^{\perp}}\left(f_{i_{0}}\right) \in \widetilde{W}_{i_{0}}$. So $f \in$ $\overline{\operatorname{span}}\left(\bigcup_{i=1}^{m} \widetilde{W}_{i}\right)$. It follows that $(\widetilde{\mathbf{W}}, \mathbf{w})$ is complete. By Corollary $7.7,(\widetilde{\mathbf{W}}, \mathbf{w})$ is a fusion frame for $\mathcal{W}$ 
Now define $V_{i}=\pi_{\mathcal{V}, \mathcal{W}^{\perp}} S_{\widetilde{\mathbf{w}}, \mathbf{w}}^{\dagger}\left(\widetilde{W}_{i}\right)$ for $i \in I$. Consider the component preserving $\widetilde{Q} \in L\left(K_{\mathcal{W}}, K_{\mathcal{V}}\right)$, given by $\widetilde{Q}\left(f_{i}\right)_{i \in I}=\left(\pi_{\mathcal{V}, \mathcal{W} \perp} S_{\widetilde{\mathbf{W}}, \mathbf{w}}^{\dagger} \pi_{\widetilde{W_{i}}} f_{i}\right)_{i \in I}$.

Let $f \in \mathcal{H}$. Since $\pi_{\widetilde{W}_{i_{0}}} \pi_{W_{i_{0}}} f=\pi_{\widetilde{W}_{i_{0}}} f$, we obtain $T_{\mathbf{V}, \mathbf{w}} \widetilde{Q} T_{\mathbf{W}, \mathbf{w}}^{*} f=\sum_{i \in I} w_{i}^{2} \pi_{\mathcal{V}, \mathcal{W} \perp} S_{\widetilde{\mathbf{W}}, \mathbf{w}}^{\dagger} \pi_{\widetilde{W}_{i}}(f)=$ $\pi_{\mathcal{V}, \mathcal{W}^{\perp}}(f)$. This shows that $(\mathbf{V}, \mathbf{w})$ is a component preserving $\widetilde{Q}$-oblique dual fusion frame of $(\mathbf{W}, \mathbf{w})$ on $\mathcal{V}$.

Note that $\widetilde{W}_{i_{0}} \subseteq W_{i_{0}}$. Assume that $\widetilde{W}_{i_{0}}=W_{i_{0}}$. Then $W_{i_{0}} \subseteq\left(\overline{\operatorname{span}}\left\{W_{i}: i \neq i_{0}\right\} \cap W_{i_{0}}\right)^{\perp}$ which is a contradiction since $W_{i_{0}} \cap \overline{\operatorname{span}}\left\{W_{i}: i \neq i_{0}\right\} \neq\{0\}$. To see that $\pi_{\mathcal{V}, \mathcal{W}^{\perp}} S_{\widetilde{\mathbf{W}}, \mathbf{w}}^{\dagger}\left(\widetilde{W}_{i_{0}}\right) \neq$ $\pi_{\mathcal{V}, \mathcal{W}^{\perp}} S_{\mathbf{W}, \mathbf{w}}^{\dagger}\left(W_{i_{0}}\right)$, take $f \in S_{\mathbf{W}, \mathbf{w}}^{\dagger}\left(W_{i_{0}} \cap \widetilde{W}_{i_{0}}^{\perp}\right), f \neq 0$. Assume, by contradiction, that $\pi_{\mathcal{V}, \mathcal{W}^{\perp}} f \in$ $\pi_{\mathcal{V}, \mathcal{W} \perp} S_{\widetilde{\mathbf{W}}, \mathbf{w}}^{\dagger}\left(\widetilde{W}_{i_{0}}\right)$. We have

$$
\pi_{\mathcal{V}, \mathcal{W}^{\perp}}(f)=\pi_{\mathcal{V}, \mathcal{W}^{\perp}} S_{\widetilde{\mathbf{W}}, \mathbf{w}}^{\dagger} S_{\widetilde{\mathbf{W}}, \mathbf{w}}(f)=\pi_{\mathcal{V}, \mathcal{W}^{\perp}} S_{\widetilde{\mathbf{W}}, \mathbf{w}}^{\dagger}\left(\sum_{i \in I, i \neq i_{0}} w_{i}^{2} \pi_{W_{i}}(f)+w_{i_{0}}^{2} \pi_{\widetilde{W}_{i_{0}}}(f)\right) .
$$

Then $\pi_{\mathcal{V}, \mathcal{W}^{\perp}} S_{\widetilde{\mathbf{W}}, \mathbf{w}}^{\dagger}\left(\sum_{i \in I, i \neq i_{0}} w_{i}^{2} \pi_{W_{i}}(f)\right) \in \pi_{\mathcal{V}, \mathcal{W}^{\perp}} S_{\widetilde{\mathbf{W}}, \mathbf{w}}^{\dagger}\left(\widetilde{W}_{i_{0}}\right)$, i.e. there exists $g \in \widetilde{W}_{i_{0}}$ such that $\pi_{\mathcal{V}, \mathcal{W} \perp} S_{\widetilde{\mathbf{W}}, \mathbf{w}}^{\dagger}\left(\sum_{i \in I, i \neq i_{0}} w_{i}^{2} \pi_{W_{i}}(f)\right)=\pi_{\mathcal{V}, \mathcal{W} \perp} S_{\widetilde{\mathbf{W}}, \mathbf{w}}^{\dagger}(g)$. Hence $S_{\widetilde{\mathbf{W}}, \mathbf{w}}^{\dagger}\left(\sum_{i \in I, i \neq i_{0}} w_{i}^{2} \pi_{W_{i}}(f)-g\right) \in \mathcal{W}^{\perp}$. But then $S_{\widetilde{\mathbf{W}}, \mathbf{w}}^{\dagger}\left(\sum_{i \in I, i \neq i_{0}} w_{i}^{2} \pi_{W_{i}}(f)-g\right)=0$ i.e. $\sum_{i \in I, i \neq i_{0}} w_{i}^{2} \pi_{W_{i}}(f)-g \in \operatorname{Ker}\left(S_{\widetilde{\mathbf{W}}, \mathbf{w}}^{\dagger}\right)=\mathcal{W}^{\perp}$ and so $\sum_{i \in I, i \neq i_{0}} w_{i}^{2} \pi_{W_{i}}(f)=g$. Then $\sum_{i \in I, i \neq i_{0}} w_{i}^{2} \pi_{W_{i}}(f) \in \widetilde{W}_{i_{0}}$. So $\sum_{i \in I, i \neq i_{0}} w_{i}^{2} \pi_{W_{i}}(f) \in$ $\left(W_{i_{0}} \cap \overline{\operatorname{span}}\left\{W_{i}: i \neq i_{0}\right\}\right)^{\perp}$ and $\sum_{i \in I, i \neq i_{0}} w_{i}^{2} \pi_{W_{i}}(f) \in W_{i_{0}} \cap \overline{\operatorname{span}}\left\{W_{i}: i \neq i_{0}\right\}$. It follows that $\sum_{i \in I, i \neq i_{0}} w_{i}^{2} \pi_{W_{i}}(f)=0$. So $f=S_{\widetilde{\mathbf{W}}, \mathbf{w}}^{\dagger} S_{\widetilde{\mathbf{W}}, \mathbf{w}}(f)=S_{\widetilde{\mathbf{W}}, \mathbf{w}}^{\dagger} w_{i_{0}}^{2} \pi_{\widetilde{W}_{i_{0}}}(f)$. Therefore $f \in S_{\widetilde{\mathbf{W}}, \mathbf{w}}^{\dagger}\left(\widetilde{W}_{i_{0}}\right) \cap$ $S_{\mathbf{W}, \mathbf{w}}^{\dagger}\left(W_{i_{0}} \cap \widetilde{W}_{i_{0}}^{\perp}\right)$, i.e. $f=S_{\widetilde{\mathbf{W}}, \mathbf{w}}^{\dagger}(h)=S_{\mathbf{W}, \mathbf{w}}^{\dagger}(s)$, where $h \in \widetilde{W}_{i_{0}}$ and $s \in W_{i_{0}} \cap \widetilde{W}_{i_{0}}^{\perp}$. But then $S_{\widetilde{\mathbf{W}}, \mathbf{w}}^{\dagger}\left(w_{i_{0}}^{2} \pi_{\widetilde{W}_{i_{0}}}\left(\frac{h}{w_{i_{0}}^{2}}\right)\right)=S_{\mathbf{W}, \mathbf{w}}^{\dagger}\left(w_{i_{0}}^{2} \pi_{W_{i_{0}}}\left(\frac{s}{w_{i_{0}}^{2}}\right)\right)$, hence $\frac{h}{w_{i_{0}}^{2}}=\frac{s}{w_{i_{0}}^{2}}$, and so $h=s=f=0$, which is a contradiction.

\section{ACKNOWLEDGEMENT}

The research of S. B. Heineken and P. M. Morillas has been partially supported by Grants PIP 112-201501-00589-CO (CONICET) and PROIPRO 3-2116 (UNSL). S. B. Heineken also acknowledges the support of Grants PICT-2011-0436 (UBA) and UBACyT 20020130100422BA.

\section{REFERENCES}

[1] P. G. Casazza and G. Kutyniok Eds., Finite Frames. Theory and Applications, Birkhäuser, Boston, 2012.

[2] P. G. Casazza and G. Kutyniok. Frames of subspaces, Contemp. Math., 345:87-113, 2004.

[3] P. G. Casazza, G. Kutyniok and S. Li. Fusion frames and distributed processing, Appl. Comput. Harmon. Anal., 25:114-132, 2008.

[4] O. Christensen, An introduction to frames and Riesz bases, Second Ed., Birkhäuser, Boston, 2016. 
[5] O. Christensen, Y.C. Eldar, Characterization of oblique dual frame pairs, EURASIP J. Appl. Signal Process., $1-11,2006$.

[6] O. Christensen and Y.C. Eldar. Oblique dual frames and shift-invariant spaces, Appl. Comput. Harmon. Anal., $17: 48-68,2004$

[7] R. J. Duffin and A. C. Schaeffer, A class of nonharmonic Fourier series, Trans. Amer. Math. Soc. 72 (1952), 341-366.

[8] Y.C. Eldar. Sampling with arbitrary sampling and reconstruction spaces and oblique dual frame vectors, J. Fourier Anal. Appl., 9(1):77-96, 2003.

[9] Y.C. Eldar. Sampling without input constrains: consistent reconstruction in arbitrary spaces, in Sampling, Wavelets and Tomography, A. Zayed and J. J. Benedetto Eds., Birkhäuser Boston, 33-60, 2003.

[10] Y.C. Eldar and T. Werther. General framework for consistent sampling in Hilbert spaces, Int. J. Wavelets Multiresolut. Inf. Process., 3(4) :497-509, 2005.

[11] S. B. Heineken, P. M. Morillas, A. M. Benavente and M. I. Zakowicz. Dual fusion frames, Arch. Math. 103: 355-365, 2014.

[12] S. B. Heineken, P. M. Morillas, Properties of finite dual fusion frames, Linear Algebra Appl. 453, 1-27, 2014.

[13] J. Kovačević and A. Chebira. An introduction to frames, Found. Trends Signal Process., 2:1-94, 2008. 